\title{
Two-phase flow equations with a dynamic capillary pressure
}

\author{
Jan Koch, Andreas Rätz, and Ben Schweizer
}

Preprint 2011-12

November 2011

Fakultät für Mathematik

Technische Universität Dortmund

Vogelpothsweg 87

44227 Dortmund 



\title{
Two-phase flow equations with a dynamic capillary pressure
}

\author{
Jan Koch, Andreas Rätz \& Ben Schweizer ${ }^{1}$
}

November 15, 2011

\begin{abstract}
We investigate the motion of two immiscible fluids in a porous medium described by the two-phase flow system. In the capillary pressure relation, we include static and dynamic hysteresis. The model is wellestablished in the context of the Richards equation, which is obtained by assuming a constant pressure for one of the two phases. We derive an existence result for this hysteresis-two-phase model for non-degenerate permeability and capillary pressure curves. A discretization scheme is introduced and numerical results for fingering experiments are obtained. The main analytical tool is a compactness result for two variables that are couled by an hysteresis relation.
\end{abstract}

key-words: two-phase flow, capillary hysteresis, finite-element scheme

subject classification: $76 \mathrm{~S} 05,76 \mathrm{Txx}, 35 \mathrm{~F} 25$

\section{Introduction}

The two-phase flow system describes the motion of two incompressible, immiscible phases in a porous medium. We consider a bounded Lipschitz domain $\Omega \subset \mathbb{R}^{n}$, occupied by the porous material, and a time interval $[0, T)$. We denote the pressures of the two fluids by $p_{1}, p_{2}: \Omega \times[0, T) \rightarrow \mathbb{R}$ and the saturation of the first fluid by $s: \Omega \times[0, T) \rightarrow \mathbb{R}$. The saturation $s=s_{1}$ is defined as the volume fraction of pore space that is filled with fluid 1, we think of the non-wetting phase. The saturation of the second fluid is $s_{2}=1-s_{1}=1-s$. Darcy's law for both velocities and conservation of mass can be combined into the system

$$
\begin{aligned}
\partial_{t} s & =\nabla \cdot\left(k_{1}(s)\left[\nabla p_{1}+g_{1}\right]\right), \\
-\partial_{t} s & =\nabla \cdot\left(k_{2}(s)\left[\nabla p_{2}+g_{2}\right]\right) .
\end{aligned}
$$

We have performed a normalization of porosity and density, the gravity vectors $g_{1}, g_{2} \in \mathbb{R}^{n}$ point in direction $-e_{n}=(0, \ldots, 0,-1) \in \mathbb{R}^{n}$. The permeabilities $k_{1}(s)=k_{1}(s(x, t), x)$ and $k_{2}(s)=k_{2}(s(x, t), x)$ are described by given functions $k_{1}, k_{2}:[0,1] \times \Omega \rightarrow[0, \infty)$. The interesting modelling problem regards the relation

\footnotetext{
${ }^{1}$ Technische Universität Dortmund, Fakultät für Mathematik, Vogelpothsweg 87, D-44227 Dortmund, Germany.
} 
between the capillary pressure $p_{1}-p_{2}$ and the saturation $s$. The simplest possibility is to assume the functional dependence $p_{1}-p_{2}=p_{c}(s)$, where $p_{c}: \mathbb{R} \rightarrow \mathbb{R}$ is the capillary pressure function.

In order to take hysteresis and dynamic effects into account, the model with an algebraic relation between $p_{1}-p_{2}$ and $s$ is replaced by

$$
p_{1}-p_{2} \in p_{c}(s)+\gamma \operatorname{sign}\left(\partial_{t} s\right)+\tau \partial_{t} s,
$$

where $\tau, \gamma \geq 0$ are two parameters and sign denotes the multi-valued function defined by $\operatorname{sign}( \pm \xi)=\{ \pm 1\}$ for $\xi>0$ and $\operatorname{sign}(0):=[-1,1]$. The model (1.3) was suggested in $[8]$ and receives considerable attention.

For $\tau>0$, the multi-valued function $\Phi: \xi \mapsto \tau \xi+\gamma \operatorname{sign}(\xi)$ can be inverted, the inverse $\Psi:=\Phi^{-1}: \mathbb{R} \rightarrow \mathbb{R}$ is a Lipschitz continuous function. With this notation, equation (1.3) transforms into

$$
\partial_{t} s=\Psi\left(p_{1}-p_{2}-p_{c}(s)\right)
$$

Our main result is an existence theorem for system (1.1)-(1.3) of partial differential equations in the case $\tau>0$. The proof is based on a compactness result that was derived in [17] for the treatment of the Richards equation. Loosely speaking, the compactness result provides the following: for every family of pressure and saturation functions that satisfies the evolution law (1.4) and the natural energy estimates, the saturation functions converge strongly along a subsequence.

Numerical results. We include a numerical treatment of the two-phase flow equations with hysteresis, (1.1)-(1.3). In agreement with the theoretical results, the finiteelement scheme turns out to be stable; this holds true even though we include spatial and temporal adaptivity. We calculate solutions in a case that corresponds to the experimental set-up which is used to observe fingering effects in porous media. The presented model and the suggested discretization scheme provide numerical solutions that show gravity fingering in porous media. A description of the scheme and the investigated parameters is presented together with numerical results in Section 3.

\subsection{Further literature on two-phase flow equations}

Unfortunately, the name "two-phase flow" is slightly ambiguous, it is used for the above system, but also for the Richards equation. The Richards equation is the simplification of the above model that is obtained by assuming that the pressure of the second phase is constant, e.g. $p_{2}=0$, and by using only (1.1) instead of the set of equations (1.1)-(1.2). Even though also this simplified model describes the motion of two fluid phases, we will use the term two-phase flow equations only when we refer to system (1.1)-(1.2).

Results on the Richards equation. Even in the case without hysteresis, i.e. with an algebraic relation $p=p_{c}(s)$ instead of (1.3), the Richards equation is an interesting mathematical object due to the possible degeneracies $k(s)=0$ for some $s$ and $p_{c}(s) \rightarrow \pm \infty$ for $s$ tending to critical saturation values. Existence results are obtained e.g. in [2] and [3], uniqueness is treated e.g. in [20] and [12], the physical 
outflow boundary conditions are treated e.g. in [1] and [23]. Regarding the numerical treatment of the Richards equation without hysteresis we mention $[4,21]$.

We want to highlight at this point the close connection between hysteresis effects and fingering in porous media; we refer to [25] and the references therein for experimental results on fingering. The analytical contributions of $[20,26]$ imply, loosely speaking, that fingering is not possible in the Richards equation without hysteresis. On the other hand, it was shown theoretically in [24] and with numerical experiments in [17] that fingering can occur in the Richards equation if hysteresis is included.

The hysteresis relation (1.3), without the coupling to a partial differential equation, poses already interesting questions regarding a functional analytic description, we refer to [28] for the corresponding discussion. In both cases, $\tau=0$ (rateindependent) and $\tau>0$ (rate-dependent), the hysteresis relation may be considered as a functional relation $s(t)=\mathcal{B}\left(t,\left.p\right|_{[0, t]}\right)$, where $\mathcal{B}$ maps the history of $p$ to a value $s(t)$, given initial values $s_{0}$. We emphasize that, even in the equilibrium situation $\partial_{t} s=0$, we cannot determine $p(t)$ from $\left.s\right|_{[0, t]}$. In this sense, the hysteresis relation cannot be inverted.

An existence result for the Richards equation with hysteresis was provided in [22] in the case that the partial differential equation is linear, i.e. in the case that $k($.$) is not depending on s$ and that $p_{c}($.$) is an affine function. In this situation, it$ was possible to treat the case $\tau=0$. Existence results for the nonlinear Richards equation with other hysteresis relations were obtained in [5] and [6] under very general assumptions, but not covering our model.

If the hysteresis model (1.3) is considered without the static part introduced by the sign function, the model can be re-written as a pseudo-parabolic equation. Using this point of view, existence results are derived in [10] and [19], including some degeneracies of the coefficients. Closest to the contribution at hand is [17], where the nonlinear Richards equation with hysteresis was studied and an existence result was derived. The compactness result of Lemma 3.3 in [17] was crucial for the Richards equations and will be used again in the work at hand.

Results on the two-phase flow equation. The two-phase flow equations without hysteresis have been studied under the aspect of existence results in [13] and [16], uniqueness and regularity issues are treated in [13] and [14], outflow conditions in [18], maximum principles appear in [16] and [18]. Physical conditions across interior interfaces are investigated in [9] and [11]. We are not aware of any contribution that derives an existence result for the two-phase flow system with hysteresis. Regarding the numerical treatment of two-phase flow without hysteresis we refer to [7].

\subsection{Assumptions and main result}

In this subsection we fix the assumptions on the coefficient functions and formulate our main result. We consider non-linear, but non-degenerate permeabilities $k_{j}, j=$ 1,2 , and a strictly increasing, non-degenerate capillary pressure curve $p_{c}$. On the relaxation constant we assume positivity, $\tau>0$. We will construct solutions of the two-phase system with the specific hysteresis relation of (1.3), but the compactness result will exploit only the more general relation (1.4). 
Initial and boundary conditions. The unknowns in the porous media model (1.1)-(1.3) are $p_{1}, p_{2}$, and $s$. Let $\Omega \subset \mathbb{R}^{n}$ such that

$\Omega$ is a Lipschitz domain and $\partial \Omega$ is decomposed as $\partial \Omega=\bar{\Gamma}_{1} \cup \bar{\Sigma}_{1}=\bar{\Gamma}_{2} \cup \bar{\Sigma}_{2}$

with $\Gamma_{j} \cap \Sigma_{j}=\emptyset$ two relatively open subsets of $\partial \Omega$ for $j=1,2$. We impose a Dirichlet condition for $p_{j}$ on $\Sigma_{j}$ and a Neumann condition for fluid $j$ on $\Gamma_{j}$, for notational convenience we impose only no-flux conditions on $\Gamma_{j}$. We assume positivity of the Hausdorff measures, $\mathcal{H}^{n-1}\left(\Sigma_{j}\right)>0$ for $j=1,2$. The Dirichlet conditions are given by two functions $p_{0,1}, p_{0,2} \in L^{2}\left(0, T ; H^{1}(\Omega)\right)$. We prescribe initial values for the saturation by a function $s_{0} \in L^{2}(\Omega)$.

Coefficient functions. Our assumptions on the coefficient functions are as follows. For six positive numbers $K_{j}, \kappa_{j}, \kappa_{j}^{0}>0, j=1,2$, we assume

$$
\begin{aligned}
& p_{c} \in C^{0,1}(\mathbb{R} \times \Omega, \mathbb{R}), \quad \gamma \in C^{0,1}(\Omega,[0, \infty)), \\
& k_{j} \in C\left(\mathbb{R} \times \Omega,\left[\kappa_{j}, \kappa_{j}^{0}\right]\right),\left\|k_{j}(x, .)\right\|_{\operatorname{Lip}(\mathbb{R}, \mathbb{R})} \leq K_{j}, \text { for } j \in\{1,2\}, x \in \Omega .
\end{aligned}
$$

We denote the Lipschitz constant of the capillary pressure by $\rho:=\left\|p_{c}\right\|_{\text {Lip }}$ and emphasize that the Lipschitz continuity of $p_{c}$ is assumed in $x$ and $s$. We will always assume $\tau>0$ and use $\Phi: \mathbb{R} \times \Omega \rightarrow \mathbb{R},(\xi, x) \mapsto \tau \xi+\gamma(x) \operatorname{sign}(\xi)$. Positivity of $\tau$ implies that $\Phi(., x)$ has a Lipschitz continuous inverse, we denote the inverse by $\Psi(., x)$. This defines $\Psi: \mathbb{R} \times \Omega \rightarrow \mathbb{R}$ with $0 \leq \partial_{\zeta} \Psi(\zeta, x) \leq 1 / \tau$ for all $\zeta \in \mathbb{R}$. We finally assume that $p_{c}$ has a positive primitive, i.e.

$$
\exists P_{c} \in C(\mathbb{R} \times \Omega, \mathbb{R}) \text { with } P_{c}(s, x) \geq 0, \partial_{s} P_{c}(s, x)=p_{c}(s, x) \text { for all } s \in \mathbb{R}, x \in \Omega \text {. }
$$

The gravity vectors $g_{1}, g_{2} \in \mathbb{R}^{n}$ are constant vectors.

Weak form of equations (1.1) and (1.2). The first two evolution equations are expressed in the usual weak form. We say that $s, p_{1}, p_{2} \in L^{2}\left(0, T ; L^{2}(\Omega)\right)$ with $\partial_{t} s \in L^{2}\left(0, T ; L^{2}(\Omega)\right)$ and $\nabla p_{1}, \nabla p_{2} \in L^{2}\left(0, T ; L^{2}(\Omega)\right)$ solve $(1.1)-(1.2)$ and the noflux condition on $\Gamma_{j}$ in the weak form if, for all test-functions $\varphi_{j} \in L^{2}\left(0, T ; H^{1}(\Omega)\right)$ with $\varphi_{j}=0$ on $\Sigma_{j}$, there holds

$$
\int_{\Omega_{T}} k_{1}(s)\left[\nabla p_{1}+g_{1}\right] \nabla \varphi_{1}+\int_{\Omega_{T}} k_{2}(s)\left[\nabla p_{2}+g_{2}\right] \nabla \varphi_{2}=-\int_{\Omega_{T}}\left(\varphi_{1}-\varphi_{2}\right) \partial_{t} s .
$$

Main Theorem. Our main result concerns the existence of solutions to the nondegenerate two-phase flow equations.

Theorem 1.1 (Existence for the two-phase flow problem with hysteresis). Let the Lipschitz domain $\Omega \subset \mathbb{R}^{n}$ with boundary parts $\Gamma_{j}$ and $\Sigma_{j}$ be as in (1.5). Let initial and boundary conditions be given by $s_{0} \in L^{2}(\Omega)$ and $p_{0,1}, p_{0,2} \in L^{2}\left(0, T ; H^{1}(\Omega)\right)$. Let the coefficients satisfy $\tau>0$ and (1.6) $-(1.8)$.

Then there exists a solution $p_{1}, p_{2} \in L^{2}\left(0, T ; H^{1}(\Omega)\right)$ and $s \in H^{1}\left(0, T ; L^{2}(\Omega)\right)$ of the two-phase hysteresis system (1.1)-(1.3). More precisely, equations (1.1), (1.2), and the no-flux condition are satisfied in the weak form (1.9), the hysteresis relation (1.3) holds pointwise almost everywhere, initial and Dirichlet boundary conditions are satisfied in the sense of traces. 


\subsection{A priori estimates and solution concept}

We start our analysis of system (1.1)-(1.3) by presenting the formal a priori estimates. These indicate the natural norms and function spaces. At the same time, we will observe a lack of spatial regularity for the saturation variable $s$. It is this lack of compactness that makes the existence proof interesting.

We multiply (1.1) with $p_{1}-p_{0,1}$ and (1.2) with $p_{2}-p_{0,2}$ and integrate over $\Omega$. Adding the equations, we obtain (1.9) with $\varphi_{j}=p_{j}-p_{0, j}$. Inserting (1.3) gives

$$
\begin{gathered}
\int_{\Omega} k_{1}(s)\left[\nabla p_{1}+g_{1}\right] \nabla\left[p_{1}-p_{0,1}\right]+\int_{\Omega} k_{2}(s)\left[\nabla p_{2}+g_{2}\right] \nabla\left[p_{2}-p_{0,2}\right] \\
=-\int_{\Omega}\left[\left(p_{1}-p_{2}\right)-p_{0,1}+p_{0,2}\right] \partial_{t} s \\
\quad \in-\int_{\Omega}\left(p_{c}(s)+\gamma \operatorname{sign}\left(\partial_{t} s\right)+\tau \partial_{t} s\right) \partial_{t} s+\int_{\Omega}\left(p_{0,1}-p_{0,2}\right) \partial_{t} s
\end{gathered}
$$

By assumption (1.8), the monotone function $p_{c}$ has a convex, positive primitive $P_{c}$. Integration over $t \in[0, T]$ and application of the Cauchy-Schwarz and the Poincaré inequality yields in the standard fashion the estimate

$$
\left.\int_{\Omega} P_{c}(s)\right|_{t=T}+\int_{\Omega_{T}}\left\{k_{1}(s)\left|\nabla p_{1}\right|^{2}+k_{2}(s)\left|\nabla p_{2}\right|^{2}+\gamma\left|\partial_{t} s\right|+\tau\left|\partial_{t} s\right|^{2}\right\} \leq C_{0},
$$

where the constant $C_{0}$ depends on the data $g_{j}, p_{0, j}, s_{0}$, on $\tau$ and on the other system constants introduced before (1.6). We exploited that $p_{c}$ is Lipschitz continuous and that, as a consequence, $P_{c}$ has at most quadratic growth in $s$. The domain of integration is $\Omega_{T}=\Omega \times(0, T)$.

\section{Variational weak solutions}

In the solution concept of Theorem 1.1 we demand that relation (1.3) holds for almost all $(x, t) \in \Omega_{T}$. In order to verify this condition, it is convenient to use additionally the notion of variational weak solutions.

Definition 1.2 (Variational weak solution). Let $\left(s, p_{1}, p_{2}\right)$ be a triple of functions with

$$
s \in L^{\infty}\left(0, T ; L^{2}(\Omega)\right), \quad \partial_{t} s \in L^{2}\left(0, T ; L^{2}(\Omega)\right), \quad p_{1}, p_{2} \in L^{2}\left(0, T ; H^{1}(\Omega)\right),
$$

satisfying, in the sense of traces, the initial condition $s=s_{0}$ on $\Omega \times\{0\}$ and the boundary conditions $p_{j}=p_{0, j}$ on $\Sigma_{j} \times(0, T)$. The triple is called a variational weak solution of the two-phase equation if the following three conditions are satisfied.

1. The evolution equations (1.1)-(1.2) and the no-flux conditions are satisfied in the weak sense of (1.9).

2. The relation $p_{1}(x, t)-p_{2}(x, t)-p_{c}(s(x, t), x)-\tau \partial_{t} s(x, t) \in[-\gamma(x), \gamma(x)]$ holds for almost every $(x, t) \in \Omega_{T}$. 
3. The variational inequality

$$
\begin{aligned}
0 & \geq \int_{\Omega_{T}}\left(p_{c}(s)-p_{0,1}+p_{0,2}\right) \partial_{t} s+\int_{\Omega_{T}}\left\{\tau\left|\partial_{t} s\right|^{2}+\gamma\left|\partial_{t} s\right|\right\} \\
& +\int_{\Omega_{T}} k_{1}(s)\left[\nabla p_{1}+g_{1}\right] \nabla\left[p_{1}-p_{0,1}\right]+\int_{\Omega_{T}} k_{2}(s)\left[\nabla p_{2}+g_{2}\right] \nabla\left[p_{2}-p_{0,2}\right]
\end{aligned}
$$

is satisfied.

Lemma 1.3. Let $\left(s, p_{1}, p_{2}\right)$ be a variational weak solution as in Definition 1.2. Then (1.3) is satisfied almost everywhere. In particular, $\left(s, p_{1}, p_{2}\right)$ is a solution of (1.1)(1.3) as described in Theorem 1.1.

Proof. We only have to show that (1.3) holds almost everywhere. For weak solutions, the two distributions $\nabla \cdot\left(k_{j}(s)\left[\nabla p_{j}+g_{j}\right]\right)= \pm \partial_{t} s$ are actually $L^{2}\left(\Omega_{T}\right)$-functions, hence we can perform an integration by parts in the last two integrals of (1.12). Then the inequality (1.12) simplifies to

$$
0 \geq \int_{\Omega_{T}}\left(p_{c}(s)-p_{1}+p_{2}\right) \partial_{t} s+\int_{\Omega_{T}}\left\{\tau\left|\partial_{t} s\right|^{2}+\gamma\left|\partial_{t} s\right|\right\}
$$

We write this as

$$
\int_{\Omega_{T}} \gamma\left|\partial_{t} s\right| \leq \int_{\Omega_{T}}\left[p_{1}-p_{2}-p_{c}(s)-\tau \partial_{t} s\right] \partial_{t} s
$$

By property 2 of variational weak solutions, the integrand on the right hand side satisfies $\left[p_{1}-p_{2}-p_{c}(., s)-\tau \partial_{t} s\right] \partial_{t} s \leq \gamma\left|\partial_{t} s\right|$ almost everywhere, and is therefore smaller or equal to the integrand on the left hand side. Since the integral inequality is in the opposite direction, the integrands must coincide, $\left[p_{1}-p_{2}-p_{c}(., s)-\tau \partial_{t} s\right] \partial_{t} s=\gamma\left|\partial_{t} s\right|$ holds almost everywhere. This, together with property 2 of variational weak solutions, implies the pointwise inclusion (1.3).

We see that Theorem 1.1 is shown once that we prove the existence of a variational weak solution as in Definition 1.2.

\section{Discrete system and proof of the main theorem}

\subsection{The discrete system}

Our next aim is to define a Galerkin scheme such that the original equations (1.1)(1.3) are approximated by a system of ordinary differential equations. With this aim we introduce a space-discretization with parameter $h>0$. We recall that three positive (and possibly small) physical parameters appear in the equations: the numbers $\kappa_{1}, \kappa_{2}>0$ are lower bounds for the permeabilities and $\tau>0$ is the time delay parameter.

In the case of a vanishing time delay, $\tau=0$, the play-type relation (1.3) can be written with the multi-valued function $\Phi^{0}(\sigma):=\gamma \operatorname{sign}(\sigma)$ as $p_{1}-p_{2} \in p_{c}(s)+\Phi^{0}\left(\partial_{t} s\right)$. 
In the general case $\tau \geq 0$ and with $\gamma=\gamma(x)$ we use $\Phi^{\tau}:=\Phi^{0}+\tau$ id, or, more precisely,

$$
\Phi^{\tau}(\sigma, x):=\left\{\begin{array}{cc}
{[-\gamma(x), \gamma(x)]} & \text { for } \quad \sigma=0 \\
\gamma(x)+\tau \sigma & \text { for } \quad \sigma>0 \\
-\gamma(x)+\tau \sigma & \text { for } \quad \sigma<0
\end{array}\right.
$$

With this choice, (1.3) can be written as $p_{1}-p_{2} \in p_{c}(s)+\Phi^{\tau}\left(\partial_{t} s\right)$; we suppress the dependence on $x$ whenever possible. We denote the inverse by $\Psi^{\tau}(., x):=\left(\Phi^{\tau}(., x)\right)^{-1}$. The inverse $\Psi^{\tau}: \mathbb{R} \times \Omega \rightarrow \mathbb{R}$ is multivalued only in the case $\tau=0$. For positive $\tau$, the function $\Psi^{\tau}$ is single-valued with maximal slope $\tau^{-1}$. In this sense, $\tau>0$ can be regarded as a regularization of the system.

Spatial discretization. We next discretize the spatial domain $\Omega$. In order to simplify notation, we describe the method for the case that the domain $\Omega$ is polygonal; in the case of a general Lipschitz domain, it poses no problem to use boundary elements that are not simplices.

Let $\mathcal{T}_{h}$ be a triangulation of $\Omega$, decomposing $\Omega$ into finitely many simplices $A \in \mathcal{T}_{h}$. Let $h>0$ be an upper bound for the diameter of all elements of $\mathcal{T}_{h}$. We denote by $\Omega_{h}=\left\{x_{1}, \ldots, x_{N}\right\}$ a suitable subset of $N$ points, such that we can associate to every triangle $A \in \mathcal{T}_{h}$ a uniquely determined point $x \in \Omega_{h} \cap A$. The set of points $\left(x_{k}\right)_{k \leq N}$ defines a projection $X^{h}: \Omega \rightarrow \Omega_{h}$. The map $X^{h}$ can also be used to define an invertible map that identifies $\mathbb{R}^{N}$ with piecewise constant functions (defined almost everywhere),

$$
J: \mathbb{R}^{N} \equiv\left\{f: \Omega_{h} \rightarrow \mathbb{R}\right\} \longrightarrow\{\hat{f}: \Omega \rightarrow \mathbb{R} \text { piecewise constant }\}=: \mathcal{P}_{0}\left(\Omega, \mathcal{T}_{h}\right),
$$

by $(J f)(x)=f\left(X^{h}(x)\right)$ for almost every $x \in \Omega$. We will furthermore use the $L^{2}(\Omega)$ orthogonal projection $P:=P_{h}: L^{2}(\Omega) \rightarrow L^{2}(\Omega)$ to the space of piecewise constant functions $\mathcal{P}_{0}\left(\Omega, \mathcal{T}_{h}\right)$. A continuous function on $\Omega$ can be discretized with the help of $X^{h}: \Omega \rightarrow \Omega_{h}$. To give an example, given $\gamma=\gamma(x)$, we can restrict to the relevant corners and consider $\left.\gamma\right|_{\Omega_{h}}$, and correspondingly the piecewise constant parameter function $\gamma^{h}(x):=J\left(\left.\gamma\right|_{\Omega_{h}}\right)(x)=\gamma\left(X^{h}(x)\right)$. Accordingly, we define the piecewise constant (in $x$ ) coefficient function $p_{c}^{h}(s, x):=p_{c}\left(s, X^{h}(x)\right)$ and its primitive $P_{c}^{h}(s, x)=P_{c}\left(s, X^{h}(x)\right)$ with $\partial_{s} P_{c}^{h}(s, x)=p_{c}^{h}(s, x)$. Analogously, the function $\Phi^{\tau}(\sigma, x)$ of $(2.1)$ is discretized in space to $\Phi_{h}^{\tau}(\sigma, x):=\Phi_{h}^{\tau}\left(\sigma, X^{h}(x)\right)$ and its inverse (in the variable $\sigma$ ) is $\Psi_{h}^{\tau}(., x)=\left(\Phi_{h}^{\tau}(., x)\right)^{-1}$. With this notation, we can now define the Galerkin scheme.

Definition 2.1 (Galerkin scheme). Our unknowns are piecewise constant functions $p_{j}^{h}: \Omega \times[0, T] \rightarrow \mathbb{R}, j=1,2$ and $s^{h}: \Omega \times[0, T] \rightarrow \mathbb{R}$, identified with maps $p_{1}^{h}, p_{2}^{h}, s^{h}:$ $[0, T] \rightarrow \mathcal{P}_{0}\left(\Omega, \mathcal{T}_{h}\right)$. We demand, for almost every $x \in \Omega$ and almost every $t \in(0, T)$,

$$
\begin{aligned}
\partial_{t} s^{h}(x, t) & =\Psi_{h}^{\tau}\left(p_{1}^{h}(x, t)-p_{2}^{h}(x, t)-p_{c}^{h}\left(s^{h}, x\right)\right), \\
s^{h}(x, 0) & =\left(P_{h} s_{0}\right)(x),
\end{aligned}
$$

where we suppressed the explicit dependence of $\Psi_{h}^{\tau}$ on $x$. The pressures $p_{1}^{h}$ and $p_{2}^{h}$ are reconstructed from $s^{h}$ as follows. We solve with two functions $\tilde{p}_{j}^{h} \in H^{1}(\Omega, \mathbb{R})$, 
$j=1,2$, in a weak sense the elliptic system

$$
\begin{aligned}
-\nabla \cdot\left(k_{1}\left(s^{h}, x\right)\left(\nabla \tilde{p}_{1}^{h}+g_{1}\right)\right) & =\Psi_{h}^{\tau}\left(p_{c}\left(s^{h}, x\right)-P_{h}\left[\tilde{p}_{1}^{h}-\tilde{p}_{2}^{h}\right]\right) \text { in } \Omega \\
-\nabla \cdot\left(k_{2}\left(s^{h}, x\right)\left(\nabla \tilde{p}_{2}^{h}+g_{2}\right)\right) & =-\Psi_{h}^{\tau}\left(p_{c}\left(s^{h}, x\right)-P_{h}\left[\tilde{p}_{1}^{h}-\tilde{p}_{2}^{h}\right]\right) \text { in } \Omega \\
\tilde{p}_{j}^{h}(\cdot, t) & =p_{0, j}(\cdot, t) \text { on } \Sigma_{j} \text { for } j=1,2,
\end{aligned}
$$

for all $t \in[0, T]$, with no-flux conditions on $\Gamma_{j}$. The discrete pressures are recovered by a projection, $p_{j}^{h}=P_{h} \tilde{p}_{j}^{h}$ for $j=1,2$.

For later use we note that the evolution equation in (2.3) can also be written as

$$
\Phi_{h}^{\tau}\left(\partial_{t} s^{h}\right)=\Phi_{h}^{0}\left(\partial_{t} s^{h}(x, t)\right)+\tau \partial_{t} s^{h} \ni p_{1}^{h}-p_{2}^{h}-p_{c}^{h}\left(s^{h}\right) .
$$

We note that $\Phi_{h}^{\tau}$ and $\Phi_{h}^{0}$ depend via $\gamma^{h}(x)$ also in a direct way on $x \in \Omega$.

\subsection{Well-posedness of the Galerkin scheme}

Our aim is to prove that (2.3) is an ordinary differential equation for $s^{h}:[0, T] \rightarrow$ $\mathcal{P}_{0}\left(\Omega, \mathcal{T}_{h}\right)$. With this perspective, we want to show that the system (2.4)-(2.6) defines a Lipschitz-continuous map $s^{h} \mapsto\left(p_{1}^{h}, p_{2}^{h}\right)=\left(P_{h} \tilde{p}_{1}^{h}, P_{h} \tilde{p}_{2}^{h}\right)$. Once this is shown, we have verified that the Galerkin scheme consists of the ordinary differential equation (2.3) (the image space $\mathcal{P}_{0}\left(\Omega, \mathcal{T}_{h}\right)$ is finite dimensional) with an intricate, but Lipschitz continuous right hand side.

The aim of the next lemma is precisely this analysis of the stationary system (2.4)-(2.6). We write $\tilde{p}_{j}^{h}=p_{0, j}+u_{j}$ for $j=1,2$, such that (2.4) and (2.5) read, omitting the $h$-dependence of the function $s$,

$$
\begin{aligned}
-\nabla \cdot\left(k_{1}(s) \nabla u_{1}\right)= & \Psi_{h}^{\tau}\left(p_{c}(s)-P_{h}\left(u_{1}-u_{2}\right)-P_{h}\left(p_{0,1}-p_{0,2}\right)\right) \\
& +\nabla \cdot\left(k_{1}(s)\left(\nabla p_{0,1}+g_{1}\right)\right), \\
-\nabla \cdot\left(k_{2}(s) \nabla u_{2}\right)= & -\Psi_{h}^{\tau}\left(p_{c}(s)-P_{h}\left(u_{1}-u_{2}\right)-P_{h}\left(p_{0,1}-p_{0,2}\right)\right) \\
& +\nabla \cdot\left(k_{2}(s)\left(\nabla p_{0,2}+g_{2}\right)\right) .
\end{aligned}
$$

We introduce some abbreviations. Let $f: \mathbb{R} \times \mathbb{R} \times \Omega \rightarrow \mathbb{R}$ be the function

$$
f(s, z, x):=-\Psi_{h}^{\tau}\left(p_{c}(s, x)-z-P_{h}\left(p_{0,1}-p_{0,2}\right)(x), x\right) .
$$

Then $s \mapsto f(s, z, x)$ is Lipschitz continuous with Lipschitz constant $\rho \tau^{-1}$. The map $z \mapsto f(s, z, x)$ is monotonically non-decreasing and Lipschitz continuous with Lipschitz constant $\tau^{-1}$. In the following, we suppress the explicit $x$-dependece of $\Psi_{h}^{\tau}$. We use $F: L^{2}(\Omega) \times L^{2}(\Omega) \rightarrow L^{2}(\Omega)$,

$$
F(s, z)(x):=f(s(x), z(x), x) .
$$

Later on, we will insert $P_{h}\left(u_{1}-u_{2}\right)$ for the variable $z$. With this choice, the expression $f(s, z, x)$ coincides, up to signs, with the first part in the right hand sides of (2.4) and (2.5). To abbreviate also the other lower order terms, we use, for $j=1,2$, the $\operatorname{map} \bar{G}_{j}: L^{2}(\Omega) \rightarrow L^{2}(\Omega)$

$$
\bar{G}_{j}(s)(x):=k_{j}(s(x), x)\left(\nabla p_{0, j}(x)+g_{j}\right) .
$$

We intend to use piecewise constant functions $s, z \in \mathcal{P}_{0}\left(\Omega, \mathcal{T}_{h}\right)$ and note that functions such as $p_{c}(s, x), F(s, z)$, or $\bar{G}_{j}(s)$ are not piecewise constant functions in general. 
Lemma 2.2 (Local existence result for the discrete stationary system). Let the data $\Omega, p_{c}, k_{j}, g_{j}, p_{0, j}$ and $\tau>0$ satisfy (1.5)-(1.8). Let the hysteresis function $\Psi_{h}^{\tau}$ and the projection $P_{h}$ be as described before Definition 2.1. Let $F$ and $\bar{G}_{j}$ be as in (2.8)(2.10). Then there exists a positive number $h_{0}>0$ such that the following statements hold.

Existence. Let $h \in\left(0, h_{0}\right)$ and $S \in L^{\infty}(\Omega)$ be arbitrary. We consider the spaces $H_{0, j}(\Omega):=\left\{u_{j} \in H^{1}(\Omega): u_{j}=0\right.$ on $\left.\Sigma_{j}\right\}$ and search for solutions $u=\left(u_{1}, u_{2}\right)$ in the product space $u \in H_{0,1}(\Omega) \times H_{0,2}(\Omega)$. For arbitrary right hand sides $G_{j} \in H_{0, j}(\Omega)^{\prime}$, $j=1,2$, there exist a unique weak solution $u=\left(u_{1}, u_{2}\right)$ of

$$
\begin{aligned}
& -\nabla \cdot\left(k_{1}(S) \nabla u_{1}\right)=-F\left(S, P_{h}\left(u_{1}-u_{2}\right)\right)+G_{1} \\
& -\nabla \cdot\left(k_{2}(S) \nabla u_{2}\right)=F\left(S,\left(P_{h}\left(u_{1}-u_{2}\right)\right)+G_{2}\right.
\end{aligned}
$$

in $\Omega$, with a weak no-flux condition $n \cdot\left[\nabla\left(p_{0, j}+u_{j}\right)+g_{j}\right]=0$ on $\Gamma_{j}$.

Lipschitz continuity. For every $R>0$ there exists a positive constant $C=C(R)$ such that the following holds. Let $s, \tilde{s} \in L^{\infty}(\Omega)$ with $\|s\|_{\infty},\|\tilde{s}\|_{\infty} \leq R$. Let $u=\left(u_{1}, u_{2}\right)$ be a solution of (2.11) for $S=s$ and $G_{j}: \varphi \mapsto-\int_{\Omega} \bar{G}_{j}(s) \nabla \varphi$. Let $\tilde{u}=\left(\tilde{u}_{1}, \tilde{u}_{2}\right)$ be a solution of (2.11) for $S=\tilde{s}$ and $G_{j}: \varphi \mapsto-\int_{\Omega} \bar{G}_{j}(\tilde{s}) \nabla \varphi$. Then

$$
\|u-\tilde{u}\|_{H^{1}\left(\Omega, \mathbb{R}^{2}\right)} \leq C\|s-\tilde{s}\|_{L^{\infty}(\Omega)} .
$$

Proof. We search for solutions in the product space $H:=H_{0,1}(\Omega) \times H_{0,2}(\Omega)$. The space $H$ is a Hilbert space with the norm of $H^{1}(\Omega) \times H^{1}(\Omega)$ and the dual space is $H^{\prime}=H_{0,1}(\Omega)^{\prime} \times H_{0,2}(\Omega)^{\prime}$.

Step 1: Re-formulation of the system. In a later step, we want to perform a continuity method. We therefore generalize the system slightly and consider, for $\lambda \in[0,1]$, the following system for $u=\left(u_{1}, u_{2}\right) \in H$,

$$
\begin{aligned}
& -\nabla \cdot\left(k_{1}(S, x) \nabla u_{1}\right)=-\lambda F\left(S, P_{h}\left(u_{1}-u_{2}\right)\right)+G_{1} \\
& -\nabla \cdot\left(k_{2}(S, x) \nabla u_{2}\right)=\lambda F\left(S, P_{h}\left(u_{1}-u_{2}\right)\right)+G_{2}
\end{aligned}
$$

in the weak form and with the same no-flux condition. With this choice of $\left(E_{\lambda}\right)$, problem $\left(E_{1}\right)$ for $\lambda=1$ coincides with the original problem (2.11). On the space $H$ we define a bilinear form $B_{S}: H \times H \rightarrow \mathbb{R}$ as

$$
B_{S}[u, \varphi]:=\int_{\Omega} k_{1}(S, x) \nabla u_{1}(x) \nabla \varphi_{1}(x)+k_{2}(S, x) \nabla u_{2}(x) \nabla \varphi_{2}(x) d x
$$

for $u=\left(u_{1}, u_{2}\right), \varphi=\left(\varphi_{1}, \varphi_{2}\right) \in H$. The pair $G:=\left(G_{1}, G_{2}\right)$ satisfies $G \in H^{\prime}$. Equation $\left(E_{\lambda}\right)$ now reads

$$
B_{S}[u, \varphi]=-\lambda \int_{\Omega} F\left(S, P_{h}\left(u_{1}-u_{2}\right)\right)\left(\varphi_{1}-\varphi_{2}\right)+\langle G, \varphi\rangle
$$

for all $\varphi \in H$; here $\langle\cdot, \cdot\rangle$ denotes the duality pairing between $H^{\prime}$ and $H$.

Step 2: A priori estimates. We use $\varphi=u$ in (2.13). Poincaré's inequality and the positiviy of $k_{1}, k_{2}$ imply the coercivity of $B_{S}$ and we obtain with $c>0$

$$
\begin{aligned}
c\|u\|_{H}^{2} & \leq \int_{\Omega} k_{1}(S)\left|\nabla u_{1}\right|^{2}+k_{2}(S)\left|\nabla u_{2}\right|^{2}=B_{S}[u, u] \\
& =-\lambda \int_{\Omega} F\left(S, P_{h}\left(u_{1}-u_{2}\right)\right)\left(u_{1}-u_{2}\right)+\langle G, u\rangle .
\end{aligned}
$$


The second term on the right hand side can be estimated directly and treated with Young's inequality as $\langle G, u\rangle \leq\|G\|_{H^{\prime}}\|u\|_{H} \leq \delta\|u\|_{H}^{2}+\delta^{-1}\|G\|_{H^{\prime}}^{2}$, for arbitrary $\delta>0$.

Concerning the integral containing $F$ we exploit the monotonicity of $f$ in $z$, furthermore the triangle inequality and Cauchy-Schwarz's inequality.

$$
\begin{aligned}
- & \int_{\Omega} F\left(S, P_{h}\left(u_{1}-u_{2}\right)\right)\left(u_{1}-u_{2}\right) \\
= & -\int_{\Omega} F\left(S, P_{h}\left(u_{1}-u_{2}\right)\right) P_{h}\left(u_{1}-u_{2}\right)+F\left(S, P_{h}\left(u_{1}-u_{2}\right)\right)\left(u_{1}-u_{2}-P_{h}\left(u_{1}-u_{2}\right)\right) \\
\leq & -\int_{\Omega} F(S, 0) P_{h}\left(u_{1}-u_{2}\right)+\left|F\left(S, P_{h}\left(u_{1}-u_{2}\right)\right)\right|\left|u_{1}-u_{2}-P_{h}\left(u_{1}-u_{2}\right)\right| \\
\leq & \|F(S, 0)\|_{L^{2}(\Omega)}\left\|P_{h}\left(u_{1}-u_{2}\right)\right\|_{L^{2}(\Omega)} \\
& \quad+\| F\left(S, P_{h}\left(u_{1}-u_{2}\right)\left\|_{L^{2}(\Omega)}\right\| u_{1}-u_{2}-P_{h}\left(u_{1}-u_{2}\right) \|_{L^{2}(\Omega)} .\right.
\end{aligned}
$$

We exploit the following properties of $P_{h}$. For $w \in H^{1}(\Omega)$ we have $\left\|P_{h} w\right\|_{L^{2}(\Omega)} \leq$ $\|w\|_{L^{2}(\Omega)}$ by Hölder's inequality, and $\left\|P_{h} w-w\right\|_{L^{2}(\Omega)} \leq C_{\mathcal{T}} h\|w\|_{H^{1}(\Omega)}$ by Poincaré's inequality for some $C_{\mathcal{T}}>0$. We use the Lipschitz continuity of $f$ in $z$ with constant $\tau^{-1}$, Young's and Poincaré's inequality to calculate

$$
\begin{aligned}
\|F(S, 0)\|_{L^{2}(\Omega)}\left\|P_{h}\left(u_{1}-u_{2}\right)\right\|_{L^{2}(\Omega)} & \\
& +\| F\left(S, P_{h}\left(u_{1}-u_{2}\right)\left\|_{L^{2}(\Omega)}\right\| u_{1}-u_{2}-P_{h}\left(u_{1}-u_{2}\right) \|_{L^{2}(\Omega)}\right. \\
\leq & \|F(S, 0)\|_{L^{2}(\Omega)}\left\|u_{1}-u_{2}\right\|_{L^{2}(\Omega)}+\frac{1}{\tau}\left\|u_{1}-u_{2}\right\|_{L^{2}(\Omega)} C_{\mathcal{T}} h\left\|u_{1}-u_{2}\right\|_{H^{1}(\Omega)} \\
& +\|F(S, 0)\|_{L^{2}(\Omega)} C_{\mathcal{T}} h\left\|u_{1}-u_{2}\right\|_{H^{1}(\Omega)} \\
\leq & \frac{C_{\mathcal{T}} h}{\tau}\left\|u_{1}-u_{2}\right\|_{H^{1}(\Omega)}^{2}+\left(1+C_{\mathcal{T}} h\right)\|F(S, 0)\|_{L^{2}(\Omega)}\left\|u_{1}-u_{2}\right\|_{H^{1}(\Omega)} \\
\leq & 2\left(\frac{C_{\mathcal{T}} h}{\tau}+\delta\left(1+C_{\mathcal{T}} h\right)^{2}\right)\|u\|_{H}^{2}+\frac{1}{\delta}\|F(S, 0)\|_{L^{2}(\Omega)}^{2},
\end{aligned}
$$

for arbitrary $\delta>0$. Choosing $\delta$ and $h_{0}$ sufficiently small, we can absorb the first term and find the a priori estimate

$$
\|u\|_{H}^{2} \leq \frac{2}{c \delta}\|F(S, 0)\|_{L^{2}(\Omega)}^{2}+C_{G}=C\left(\|S\|_{L^{\infty}(\Omega)},\|G\|_{H^{\prime}}\right) .
$$

Estimate (2.14) holds for every $h \in\left(0, h_{0}\right)$ and we emphasize that the number $h_{0}$ is independent of the data $S$ and $G_{j}$.

Step 3: The continuity method. We define the set

$$
\Lambda:=\left\{\lambda \in[0,1] \mid \forall\left(G_{1}, G_{2}\right) \in H^{\prime} \text { there exists a unique solution } u \in H \text { of }\left(E_{\lambda}\right)\right\},
$$

with the aim to show that $\Lambda$ contains $\lambda=1$. It is an immediate observation that $\Lambda$ contains $\lambda=0$. Indeed, equation $\left(E_{\lambda}\right)$ for $\lambda=0$ consists of two decoupled linear elliptic equations that can be solved uniquely with the Lax-Milgram theorem.

We will show the following Claim: For every $\lambda_{0} \in \Lambda$, there exists $\varepsilon>0$ independent of $\lambda_{0}$, such that $\left(E_{\lambda}\right)$ has a unique solution for all $\lambda \in\left(\lambda_{0}, \lambda_{0}+\varepsilon\right)$. Once that the claim is verified, we can apply it a finite number of times and obtain $1 \in \Lambda$ and thus the existence and uniqueness result. 
In order to prove the claim we use a fixed point method. We define an iteration by considering, for given $\tilde{u}=\left(\tilde{u}_{1}, \tilde{u}_{2}\right) \in H$, the following equation for $u=\left(u_{1}, u_{2}\right)$,

$$
\begin{aligned}
B_{S}[u, \varphi]= & -\lambda_{0} \int_{\Omega} F\left(S, P_{h}\left(u_{1}-u_{2}\right)\right)\left(\varphi_{1}-\varphi_{2}\right) \\
& -\varepsilon \int_{\Omega} F\left(S, P_{h}\left(\tilde{u}_{1}-\tilde{u}_{2}\right)\right)\left(\varphi_{1}-\varphi_{2}\right)+\langle G, \varphi\rangle
\end{aligned}
$$

for all $\varphi=\left(\varphi_{1}, \varphi_{2}\right) \in H$. Since $\lambda_{0}$ is an element of $\Lambda$, by definition of $\Lambda$, we find a unique solution $\left(u_{1}, u_{2}\right)$ of $(2.15)$. We exploit here that the vector $G-\varepsilon\left(-F\left(S, P_{h}\left(\tilde{u}_{1}-\right.\right.\right.$ $\left.\left.\left.\tilde{u}_{2}\right)\right), F\left(S, P_{h}\left(\tilde{u}_{1}-\tilde{u}_{2}\right)\right)\right)$ is an element of $H^{\prime}$. The unique solvability property defines an operator $T: H \rightarrow H, T(\tilde{u})=u$. We note that a fixed-point $\left(u_{1}, u_{2}\right)=\left(\tilde{u}_{1}, \tilde{u}_{2}\right)$ provides a solution of $\left(E_{\lambda}\right)$ for $\lambda=\lambda_{0}+\varepsilon$.

It therefore suffices to show that the map $T$ as above is contractive for $\varepsilon>$ 0 sufficiently small (the smallness must be independent of $\lambda_{0}$ ). Let $\tilde{u}=\left(\tilde{u}_{1}, \tilde{u}_{2}\right)$, $\tilde{v}=\left(\tilde{v}_{1}, \tilde{v}_{2}\right) \in H$ be different data, we consider solutions $T(\tilde{u})=u=\left(u_{1}, u_{2}\right)$ and $T(\tilde{v})=v=\left(v_{1}, v_{2}\right)$. We investigate (2.15) for $u$ and $v$, subtract both equations and set $\varphi=u-v \in H$, i.e. $\varphi_{1}=u_{1}-v_{1}$ and $\varphi_{2}=u_{2}-v_{2}$. We find, for some $c>0$,

$$
\begin{aligned}
c \| u & -v \|_{H}^{2} \leq B_{S}[u-v, u-v] \\
= & -\lambda_{0} \int_{\Omega}\left[F\left(S, P_{h}\left(u_{1}-u_{2}\right)\right)-F\left(S, P_{h}\left(v_{1}-v_{2}\right)\right)\right]\left(\left(u_{1}-v_{1}\right)-\left(u_{2}-v_{2}\right)\right) \\
& -\varepsilon \int_{\Omega}\left[F\left(S, P_{h}\left(\tilde{u}_{1}-\tilde{u}_{2}\right)\right)-F\left(S, P_{h}\left(\tilde{v}_{1}-\tilde{v}_{2}\right)\right)\right]\left(\left(u_{1}-v_{1}\right)-\left(u_{2}-v_{2}\right)\right) .
\end{aligned}
$$

In the first integral we apply the monotonicity and Lipschitz continuity of $f$ in $z$, Cauchy-Schwarz and the properties of the projection $P_{h}$. We obtain

$$
\begin{aligned}
- & \lambda_{0} \int_{\Omega}\left[F\left(S, P_{h}\left(u_{1}-u_{2}\right)\right)-F\left(S, P_{h}\left(v_{1}-v_{2}\right)\right)\right]\left(\left(u_{1}-v_{1}\right)-\left(u_{2}-v_{2}\right)\right) \\
\leq & \int_{\Omega}\left|F\left(S, P_{h}\left(u_{1}-u_{2}\right)\right)-F\left(S, P_{h}\left(v_{1}-v_{2}\right)\right)\right| \cdot \\
& \cdot\left|u_{1}-u_{2}-\left(v_{1}-v_{2}\right)-P_{h}\left(\left(u_{1}-u_{2}\right)-\left(v_{1}-v_{2}\right)\right)\right| \\
& \leq \frac{2}{\tau}\left\|P_{h}(u-v)\right\|_{L^{2}\left(\Omega, \mathbb{R}^{2}\right)}\left\|u-v-P_{h}(u-v)\right\|_{L^{2}\left(\Omega, \mathbb{R}^{2}\right)} \leq \frac{2}{\tau} C_{\mathcal{T}} h\|u-v\|_{H}^{2} .
\end{aligned}
$$

The second integral is treated with the Lipschitz continuity of $f$ in $z$,

$$
\begin{gathered}
-\varepsilon \int_{\Omega}\left[F\left(S, P_{h}\left(\tilde{u}_{1}-\tilde{u}_{2}\right)\right)-F\left(S, P_{h}\left(\tilde{v}_{1}-\tilde{v}_{2}\right)\right)\right]\left(\left(u_{1}-v_{1}\right)-\left(u_{2}-v_{2}\right)\right) \\
\leq \varepsilon \frac{2}{\tau}\|\tilde{u}-\tilde{v}\|_{H}\|u-v\|_{H} \leq\left(\frac{\varepsilon}{\tau}\right)^{2} \frac{1}{\delta}\|\tilde{u}-\tilde{v}\|_{H}^{2}+\delta\|u-v\|_{H}^{2},
\end{gathered}
$$

for arbitrary $\delta>0$. Summarizing, we obtained

$$
c\|u-v\|_{H}^{2} \leq \frac{2}{\tau} C_{\mathcal{T}} h\|u-v\|_{H}^{2}+\left(\frac{\varepsilon}{\tau}\right)^{2} \frac{1}{\delta}\|\tilde{u}-\tilde{v}\|_{H}^{2}+\delta\|u-v\|_{H}^{2} .
$$

We choose $h_{0}>0$ and $\delta>0$ small to absorb the first and the third term. These choices depend on $\tau$ and the lower bounds for the permeabilities, but they are independent of 
$S$ and $\lambda_{0}$. For sufficiently small $\varepsilon$ we obtain the contraction property of $T$. Using the special argument $\tilde{u}=0$ and the solution $T(0)$ we find that $T$ maps a sufficiently large ball into itself. The Banach fixed point theorem yields the existence of a solution in this ball. The fixed point is globally unique, since the $T$ is contractive on any ball. This provides that for such $\varepsilon>0$ the equation $\left(E_{\lambda}\right)$ has a unique solution for $\lambda<\lambda_{0}+\varepsilon$ and hence the claim.

Step 4: Proof of the Lipschitz estimate (2.12). In contrast to the previous steps, we now investigate how variations of the parameter function $S$ effect solutions. With this aim, let $u$ be a solution of (2.11) for $S=s \in L^{\infty}(\Omega)$ with $G_{j}=\nabla \cdot \bar{G}_{j}(s)$ in the sense of $G_{j}(s): \varphi \mapsto-\int_{\Omega} \bar{G}_{j}(s) \nabla \varphi$ for $\varphi \in H_{0, j}(\Omega)$, where $\bar{G}_{j}$ is as in (2.10). For other saturation data $\tilde{s} \in L^{\infty}(\Omega)$, let $\tilde{u}$ be the solution of $(2.11)$ for $S=\tilde{s}$ with $G_{j}=\nabla \cdot \bar{G}_{j}(\tilde{s})$.

In order to compare $u$ with $\tilde{u}$, we choose $\varphi=u-\tilde{u}$ as a test-function in both variants of equation (2.11). We calculate

$$
\begin{aligned}
c \| u & -\tilde{u} \|_{H}^{2} \leq B_{s}[u-\tilde{u}, u-\tilde{u}]=\int_{\Omega} k_{1}(s)\left|\nabla\left(u_{1}-\tilde{u}_{1}\right)\right|^{2}+k_{2}(s)\left|\nabla\left(u_{2}-\tilde{u}_{2}\right)\right|^{2} \\
= & \sum_{j=1}^{2} \int_{\Omega}\left(k_{j}(\tilde{s})-k_{j}(s)\right) \nabla \tilde{u}_{j} \cdot \nabla\left(u_{j}-\tilde{u}_{j}\right) \\
& -\int_{\Omega}\left[F\left(s, P_{h}\left(u_{1}-u_{2}\right)\right)-F\left(\tilde{s}, P_{h}\left(\tilde{u}_{1}-\tilde{u}_{2}\right)\right)\right]\left(\left(u_{1}-\tilde{u}_{1}\right)-\left(u_{2}-\tilde{u}_{2}\right)\right) \\
& +\sum_{j=1}^{2} \int_{\Omega}\left(\bar{G}_{j}(s)-\bar{G}_{j}(\tilde{s})\right) \nabla\left(u_{j}-\tilde{u}_{j}\right) .
\end{aligned}
$$

We next use the a priori estimate (2.14), providing $\|\tilde{u}\|_{H} \leq C_{a p}(R)$ for some constant $C_{a p}(R)>0$. Hence the first sum on the right hand side can be estimated exploiting the Lipschitz continuity of $k_{1}$ and $k_{2}$, Poincaré's and Young's inequality.

$$
\begin{aligned}
& \sum_{j=1}^{2} \int_{\Omega}\left(k_{j}(\tilde{s})-k_{j}(s)\right) \nabla \tilde{u}_{j} \cdot \nabla\left(u_{j}-\tilde{u}_{j}\right) \\
& \quad \leq L_{k}\|s-\tilde{s}\|_{L^{\infty}(\Omega)}\|\tilde{u}\|_{H}\|u-\tilde{u}\|_{H} \leq L_{k}^{2} \frac{C_{a p}(R)^{2}}{\delta}\|s-\tilde{s}\|_{L^{\infty}(\Omega)}^{2}+\delta\|u-\tilde{u}\|_{H}^{2}
\end{aligned}
$$

for $L_{k}>0$, which depends on the Lipschitz constants of $k_{1}$ and $k_{2}$. We can choose $\delta>0$ sufficiently small to absorb the last term into the left hand side.

The second integral on the right hand side of (2.16) is treated with the monotonicity of $f$ in $z$. We furthermore use the Lipschitz continuity of $f$ in $s$ and $z$ and the properties of the $L^{2}$-orthogonal projection $P_{h}$.

$$
\begin{aligned}
- & \int_{\Omega}\left[F\left(s, P_{h}\left(u_{1}-u_{2}\right)\right)-F\left(\tilde{s}, P_{h}\left(\tilde{u}_{1}-\tilde{u}_{2}\right)\right)\right]\left(\left(u_{1}-\tilde{u}_{1}\right)-\left(u_{2}-\tilde{u}_{2}\right)\right) \\
= & -\int_{\Omega}\left[F\left(s, P_{h}\left(u_{1}-u_{2}\right)\right)-F\left(\tilde{s}, P_{h}\left(u_{1}-u_{2}\right)\right)\right]\left(\left(u_{1}-\tilde{u}_{1}\right)-\left(u_{2}-\tilde{u}_{2}\right)\right) \\
& -\int_{\Omega}\left[F\left(\tilde{s}, P_{h}\left(u_{1}-u_{2}\right)\right)-F\left(\tilde{s}, P_{h}\left(\tilde{u}_{1}-\tilde{u}_{2}\right)\right)\right]\left(\left(u_{1}-\tilde{u}_{1}\right)-\left(u_{2}-\tilde{u}_{2}\right)\right)
\end{aligned}
$$




$$
\begin{aligned}
\leq & L_{f}\|s-\tilde{s}\|_{L^{\infty}(\Omega)} C_{a p}(R)\|u-\tilde{u}\|_{H} \\
& +\int_{\Omega}\left|F\left(\tilde{s}, P_{h}\left(u_{1}-u_{2}\right)\right)-F\left(\tilde{s}, P_{h}\left(\tilde{u}_{1}-\tilde{u}_{2}\right)\right)\right| \cdot \\
& \quad \cdot\left|\left(u_{1}-\tilde{u}_{1}\right)-\left(u_{2}-\tilde{u}_{2}\right)-P_{h}\left(\left(u_{1}-\tilde{u}_{1}\right)-\left(u_{2}-\tilde{u}_{2}\right)\right)\right| \\
\leq & \frac{L_{f}^{2}}{\delta} C_{a p}(R)^{2}\|s-\tilde{s}\|_{L^{\infty}(\Omega)}^{2}+\delta\|u-\tilde{u}\|_{H}^{2}+\frac{1}{\tau} C_{\mathcal{T}} h\|u-\tilde{u}\|_{H}^{2},
\end{aligned}
$$

for arbitrary $\delta>0$, where $L_{f}>0$ depends on the Lipschitz constant of $f$ in $s$. Choosing $h_{0}, \delta>0$ sufficiently small, we can absorb the last two terms into the left hand side. Once more, the choice of $h_{0}$ and $\delta$ is independent of $s, \tilde{s}$, and $R$.

The estimate of the last integral on the right hand side of (2.16) exploits the Lipschitz continuity of $k_{j}$,

$$
\begin{aligned}
& \sum_{j=1}^{2} \int_{\Omega}\left(\bar{G}_{j}(s)-\bar{G}_{j}(\tilde{s})\right) \nabla\left(u_{j}-\tilde{u}_{j}\right) \\
& \quad \leq \sum_{j=1}^{2} \int_{\Omega}\left|k_{j}(s)-k_{j}(\tilde{s})\right|\left|\nabla p_{0, j}+g_{j}\right|\left|\nabla\left(u_{j}-\tilde{u}_{j}\right)\right| \\
& \quad \leq C L_{k}\|s-\tilde{s}\|_{L^{\infty}(\Omega)}\|u-\tilde{u}\|_{H} \leq \delta\|u-\tilde{u}\|_{H}^{2}+C^{2} \frac{L_{k}^{2}}{\delta}\|s-\tilde{s}\|_{L^{\infty}(\Omega)}^{2},
\end{aligned}
$$

where $C$ depends only on the data $p_{0, j}$ and $g_{j}$ for $j=1,2$. Once more, we can choose $\delta>0$ sufficiently small to absorb the first term.

We conclude as follows. We insert the three intermediate estimates into (2.16), choose $\delta, h_{0}>0$ sufficiently small, and absorb terms containing $\|u-\tilde{u}\|_{H}^{2}$ into the left hand side. As result, we obtain (2.12).

Remark 2.3. The Lipschitz continuity of $k_{j}$ in $s$ was not used in the existence part of the above lemma. Furthermore, the above proof is not restricted to our special choice of the non-linearity $\Psi_{h}^{\tau}$. The essential properties are the Lipschitz continuity of $\Psi_{h}^{\tau}$ and the fact that $\Psi_{h}^{\tau}(\cdot, x)$ is monotonically nondecreasing for every $x \in \Omega$. The special choice of $\Psi_{h}^{\tau}$ is used later in the compactness Lemma 2.5 and it was used in Lemma 1.3.

With Lemma 2.2, we have shown that (2.3) is an ordinary differential equations with Lipschitz continuous right hand side in $\mathcal{P}_{0}\left(\Omega, \mathcal{T}_{h}\right)$ for every $t$. Hence a local solution of the Galerkin scheme of Definition 2.1 exists. Additionally, as a consequence of the general theory of ordinary differential equations, we know the following: if we can show that the norm $\|s(t)\|_{\infty}$ is bounded for every solution $s$ on an arbitrary time interval $(0, T)$, with a bound that is independent of $T$, then the solution can be extended and exists for all times.

In the next subsection we will derive such a uniform bound and thus obtain, in particular, the global existence of solutions to the Galerkin scheme.

\subsection{A priori estimates for the time-dependent system}

We intend to perform the limit $h \rightarrow 0$ for the solutions $s^{h}, \tilde{p}_{1}^{h}, \tilde{p}_{2}^{h}$ of the Galerkin scheme of Definition 2.1. In a first step, we derive $h$-independent estimates for such 
solutions of the time-dependent system. The two lemmas 2.4 and 2.7 below are very similar to results of [17], they are essentially adaptations to the two-phase flow system. The two proofs follow a standard scheme.

Lemma 2.4 (Energy estimates). Let the coefficient functions, initial and boundary data be given as in Lemma 2.2 and $s_{0} \in L^{2}(\Omega)$. Then there exists a number $C>0$, independent of $h>0$ and $T>0$, such that every solution $\tilde{p}_{1}^{h}, \tilde{p}_{2}^{h}, s^{h}$ to the Galerkin scheme of Definition 2.1 satisfies the uniform bound

$$
\left\|\tilde{p}_{1}^{h}\right\|_{L^{2}\left(0, T ; H^{1}(\Omega)\right)}^{2}+\left\|\tilde{p}_{2}^{h}\right\|_{L^{2}\left(0, T ; H^{1}(\Omega)\right)}^{2}+\left\|\partial_{t} s^{h}\right\|_{L^{2}\left(0, T ; L^{2}(\Omega)\right)}^{2} \leq C .
$$

Proof. We abbreviate the pressure differences as $\tilde{p}^{h}:=\tilde{p}_{1}^{h}-\tilde{p}_{2}^{h}$ and $p^{h}:=p_{1}^{h}-p_{2}^{h}=$ $P_{h} \tilde{p}^{h}$. Concerning the boundary data, we use $p_{0}:=p_{0,1}-p_{0,2}$.

We start by writing the Galerkin evolution equation in a form that is similar to the continuous formulation (1.1)-(1.2). We write (2.3) and (2.4) as

$$
\begin{aligned}
& \partial_{t} s^{h}-\nabla \cdot\left(k_{1}\left(s^{h}, x\right)\left(\nabla \tilde{p}_{1}^{h}+g_{1}\right)\right) \\
& \quad=-\Psi_{h}^{\tau}\left(p_{c}^{h}\left(s^{h}, x\right)-p_{1}^{h}+p_{2}^{h}\right)+\Psi_{h}^{\tau}\left(p_{c}\left(s^{h}, x\right)-p_{1}^{h}+p_{2}^{h}\right),
\end{aligned}
$$

and write (2.3) and (2.5) as

$$
\begin{aligned}
& -\partial_{t} s^{h}-\nabla \cdot\left(k_{2}\left(s^{h}, x\right)\left(\nabla \tilde{p}_{2}^{h}+g_{2}\right)\right) \\
& \quad=\Psi_{h}^{\tau}\left(p_{c}^{h}\left(s^{h}, x\right)-p_{1}^{h}+p_{2}^{h}\right)-\Psi_{h}^{\tau}\left(p_{c}\left(s^{h}, x\right)-p_{1}^{h}+p_{2}^{h}\right) .
\end{aligned}
$$

We multiply (2.18) with $\tilde{p}_{1}^{h}-p_{0,1}$ and $(2.19)$ with $\tilde{p}_{2}^{h}-p_{0,2}$ and integrate over $\Omega$. Summing up the resulting equations yields

$$
\begin{gathered}
\int_{\Omega} \partial_{t} s^{h}\left(\tilde{p}^{h}-p_{0}\right) d x+\sum_{j=1}^{2} \int_{\Omega} k_{j}\left(s^{h}, x\right)\left(\nabla \tilde{p}_{j}^{h}+g_{j}\right)\left(\nabla \tilde{p}_{j}^{h}-\nabla p_{0, j}\right) d x \\
=-\int_{\Omega}\left[\Psi_{h}^{\tau}\left(p_{c}^{h}\left(s^{h}, x\right)-p^{h}\right)-\Psi_{h}^{\tau}\left(p_{c}\left(s^{h}, x\right)-p^{h}\right)\right]\left(\tilde{p}^{h}-p_{0}\right) d x .
\end{gathered}
$$

The space derivatives on the left hand side provide a positive term,

$$
\int_{\Omega} k_{j}\left(s^{h}, x\right)\left(\nabla \tilde{p}_{j}^{h}+g_{j}\right) \nabla \tilde{p}_{j}^{h} \geq \frac{\kappa_{j}}{2}\left\|\nabla \tilde{p}_{j}^{h}\right\|_{L^{2}(\Omega)}^{2}-C_{j}^{1}
$$

where $C_{j}^{1}, j=1,2$, depend on the bounds $\kappa_{j}$ and $\kappa_{j}^{0}$ of the permeabilities and on the gravity vectors $g_{j}$.

The time derivative on the left hand side of (2.20) is treated with the hysteresis differential equation (2.7), which reads

$$
\Phi_{h}^{0}\left(\partial_{t} s^{h}(x, t)\right)+\tau \partial_{t} s^{h}(x, t)+p_{c}^{h}\left(s^{h}, x\right) \ni p^{h}(x, t) .
$$

Using the monotonicity $\Phi_{h}^{0}(\xi) \xi \geq 0$ for all $\xi \in \mathbb{R}$, we can calculate with the primitive $P_{c}^{h}(\cdot, x)$ of $p_{c}^{h}(\cdot, x)$, exploiting that $P_{h}$ is an $L^{2}(\Omega)$-orthogonal projection,

$$
\int_{\Omega} \partial_{t} s^{h} \tilde{p}^{h}=\int_{\Omega} \partial_{t} s^{h} p^{h} \geq \int_{\Omega} \tau\left|\partial_{t} s^{h}\right|^{2}+p_{c}^{h}\left(s^{h}\right) \partial_{t} s^{h}=\tau\left\|\partial_{t} s^{h}\right\|_{L^{2}(\Omega)}^{2}+\partial_{t} \int_{\Omega} P_{c}^{h}\left(s^{h}, x\right) .
$$


We have assumed that $P_{c}$ and thus $P_{c}^{h}$ can be chosen as positive functions. We have therefore recognized three relevant positive terms on the left hand side of (2.20).

Concerning the remaining integrals on the left hand side of (2.20) we calculate

$$
\begin{aligned}
& \left|\int_{\Omega} \partial_{t} s^{h} p_{0}\right|+\sum_{j=1}^{2}\left|\int_{\Omega} k_{j}\left(s^{h}\right)\left(\nabla \tilde{p}_{j}^{h}+g_{j}\right) \nabla p_{0, j}\right| \\
& \quad \leq \frac{\tau}{2}\left\|\partial_{t} s^{h}\right\|_{L^{2}(\Omega)}^{2}+\frac{\kappa_{1}}{4}\left\|\nabla \tilde{p}_{1}^{h}\right\|_{L^{2}(\Omega)}^{2}+\frac{\kappa_{2}}{4}\left\|\nabla \tilde{p}_{2}^{h}\right\|_{L^{2}(\Omega)}^{2}+C_{1}^{2}+C_{2}^{2},
\end{aligned}
$$

where the constants $C_{j}^{2}, j=1,2$, depend on $\kappa_{j}$ and $\kappa_{j}^{0}$, on the data $p_{0, j}$ (directly and through $\left.p_{0}=p_{0,1}-p_{0,2}\right)$, and on the gravity vectors $g_{j}$.

It remains to treat the term on the right hand side of (2.20). Exploiting the Lipschitz continuity of $p_{c}$ in $x$ with constant $\rho$ we find

$$
\left|\left[\Psi_{h}^{\tau}\left(p_{c}^{h}\left(x, s^{h}\right)-p^{h}\right)-\Psi_{h}^{\tau}\left(p_{c}\left(x, s^{h}\right)-p^{h}\right)\right]\right| \leq \frac{\rho}{\tau} h,
$$

and the corresponding product is treated with the Cauchy-Schwarz inequality.

Summarizing, we find

$$
\begin{gathered}
\frac{\kappa_{1}}{4}\left\|\nabla \tilde{p}_{1}^{h}\right\|_{L^{2}(\Omega)}^{2}+\frac{\kappa_{2}}{4}\left\|\nabla \tilde{p}_{2}^{h}\right\|_{L^{2}(\Omega)}^{2}+\frac{\tau}{2}\left\|\partial_{t} s^{h}\right\|_{L^{2}(\Omega)}^{2}+\partial_{t} \int_{\Omega} P_{c}^{h}\left(s^{h}, x\right) d x \\
\leq C_{1}^{1}+C_{2}^{1}+C_{1}^{2}+C_{2}^{2}+\frac{\rho h}{\tau}\left(\left\|\tilde{p}^{h}\right\|_{L^{2}(\Omega)}^{2}+\left\|p_{0}\right\|_{L^{2}(\Omega)}^{2}\right) .
\end{gathered}
$$

For sufficiently small $h$, depending only on coefficient and boundary data, we can absorb the term containing $\left\|\tilde{p}^{h}\right\|_{L^{2}(\Omega)}^{2}$. An integration over $(0, T)$ provides the estimate (2.17). The constant $C$ depends on the coefficient and boundary data and, additionally, on the $L^{2}$-norm of the initial data, $\left\|s_{0}\right\|_{L^{2}(\Omega)}$, since the integral $\int_{\Omega} P_{c}^{h}\left(P_{h} s_{0}\right)$ enters the estimate.

\subsection{Compactness}

We are now in a position to apply a compactness result that has been developed in the context of the Richards equation. Lemma 3.3 of [17] concludes from uniform estimates for the family $\tilde{p}^{h}$ and the hysteresis relation for $s^{h}$ an $L^{2}\left(\Omega_{T}\right)$-compactness result for the sequence $s^{h}$. The precise statement is as follows.

Lemma 2.5 ([17, Lemma 3.3], Regularity and compactness from the hysteresis relation). Let $s^{h}$ and $\tilde{p}^{h}$ satisfy the ordinary differential equation of the hysteresis relation

$$
\begin{aligned}
\partial_{t} s^{h}\left(x_{k}, t\right) & =-\Psi_{\delta, h}^{\tau}\left(p_{c}\left(x_{k}, s^{h}\right)-p^{h}\left(x_{k}, t\right)\right) \quad \forall x_{k} \in \Omega_{h} \\
s^{h}\left(x_{k}, 0\right) & =P_{h} s_{0}\left(x_{k}\right)
\end{aligned}
$$

for $p^{h}=P_{h} \tilde{p}^{h}$. Let $q \in[1, \infty]$ be a number and let $s_{0} \in L^{q}(\Omega)$ define initial values. Then there holds an estimate

$$
\left\|\partial_{t} s^{h}\right\|_{L^{2}\left(0, T ; L^{q}(\Omega)\right)}+\left\|s^{h}\right\|_{L^{2}\left(0, T ; L^{q}(\Omega)\right)} \leq C\left\|\tilde{p}^{h}\right\|_{L^{2}\left(0, T ; L^{q}(\Omega)\right)},
$$

where the constant $C$ does not depend on $h$ and $\delta$. 
Let additionally the following estimate hold with $C$ independent of $h$ and $\delta$,

$$
\left\|\tilde{p}^{h}\right\|_{L^{2}\left(0, T ; H^{1}(\Omega)\right)} \leq C
$$

Then the family $s^{h}$ is pre-compact in the space $L^{2}(\Omega \times(0, T))$.

Regarding our application of the lemma we remark the following. (i) We use Lemma 2.5 with $\delta=0$. The nonlinear function $\Psi_{\delta, h}^{\tau}=\Psi_{0, h}^{\tau}$ of Lemma 2.5 then coincides with our function $\Psi_{h}^{\tau}$. (ii) Our non-linear function $p_{c}$ satisfies the conditions that have been imposed for Lemma 2.5. (iii) The projection $P_{h}$ is as in the present contribution. (iv) We apply Lemma 2.5 to $p^{h}:=p_{1}^{h}-p_{2}^{h}$ and $\tilde{p}^{h}:=\tilde{p}_{1}^{h}-\tilde{p}_{2}^{h}$, emphasizing that, by linearity, also $P_{h} \tilde{p}^{h}=p^{h}$ is satisfied. With this setting, the hysteresis equation of the lemma is identical to our equation (2.3).

Lemma 2.6 (Compactness of the family of saturations). Let the coefficient functions, initial and boundary data be as in Lemma 2.2 and $s_{0} \in L^{2}(\Omega)$. Let $\tilde{p}_{1}^{h}, \tilde{p}_{2}^{h}, s^{h}$ be a family of solutions to the Galerkin scheme of Definition 2.1 for a sequence $h \rightarrow 0$. Then

$$
\text { the sequence } s^{h} \text { is pre-compact in } L^{2}(\Omega \times(0, T)) \text {. }
$$

Proof. By the above remarks, we can apply Lemma 2.5, to the pressure difference $\tilde{p}^{h}:=\tilde{p}_{1}^{h}-\tilde{p}_{2}^{h}$. We use the integrability exponent $q=2$. The a priori estimates of Lemma 2.4 provide the boundedness

$$
\left\|\tilde{p}^{h}\right\|_{L^{2}\left(0, T ; H^{1}(\Omega)\right)}^{2} \leq 2\left(\left\|\tilde{p}_{1}^{h}\right\|_{L^{2}\left(0, T ; H^{1}(\Omega)\right)}^{2}+\left\|\tilde{p}_{2}^{h}\right\|_{L^{2}\left(0, T ; H^{1}(\Omega)\right)}^{2}\right) \leq C
$$

with $C$ independent of $h$. This shows that (2.22) is satisfied. We can apply the second part of Lemma 2.5 and conclude (2.23).

\subsection{Limit procedure $h \rightarrow 0$}

We consider now limit functions to the solution sequence $\left(s^{h}, \tilde{p}_{1}^{h}, \tilde{p}_{2}^{h}\right)$ for $h \rightarrow 0$. Due to the uniform estimates of Lemma 2.4 we find a subsequence $h \rightarrow 0$ and limit functions $s, p_{1}, p_{2}$ such that

$$
\begin{array}{rll}
\tilde{p}_{j}^{h} \rightarrow p_{j} & \text { in } & L^{2}\left(0, T ; H^{1}(\Omega)\right) \text { for } j=1,2, \\
s^{h} \rightarrow s, \partial_{t} s^{h} \rightarrow \partial_{t} s & \text { in } & L^{2}\left(0, T ; L^{2}(\Omega)\right) .
\end{array}
$$

Furthermore, by the compactness result of (2.23) we find the strong convergence

$$
s^{h} \rightarrow s \quad \text { in } \quad L^{2}\left(0, T ; L^{2}(\Omega)\right)
$$

The following lemma concludes the proof of Theorem 1.1.

Lemma 2.7. Let the data be as in Lemma 2.2 and $s_{0} \in L^{2}(\Omega)$. Let $\tilde{p}_{1}^{h}, \tilde{p}_{2}^{h}, s^{h}$ be a family of solutions to the Galerkin scheme of Definition 2.1 for a sequence $h \rightarrow 0$. For a subsequence, let $p_{1}, p_{2}$, and $s$ be limit functions as in (2.24)-(2.26). Then the limit triple $\left(s, p_{1}, p_{2}\right) \in L^{2}\left(0, T ; L^{2}(\Omega)\right) \times L^{2}\left(0, T ; H^{1}\left(\Omega, \mathbb{R}^{2}\right)\right)$ is a variational weak solution according to Definition 1.2. 
Proof. The limit functions are contained in the function spaces as described in (1.11). The weak convergence allows to take limits in the initial and boundary conditions, hence they are satisfied by the limit functions. We have to check the three items of Definition 1.2. In the calculations below we use once more $p^{h}=p_{1}^{h}-p_{2}^{h}, \tilde{p}^{h}=\tilde{p}_{1}^{h}-\tilde{p}_{2}^{h}$, and $p_{0}=p_{0,1}-p_{0,2}$.

Item 1. We have to show (1.1)-(1.2), i.e. that $\partial_{t} s=\nabla \cdot\left(k_{1}(s)\left[\nabla p+g_{1}\right]\right)$ and $\partial_{t} s=-\nabla \cdot\left(k_{2}(s)\left[\nabla p+g_{2}\right]\right)$ is satisfied in the weak sense with the no-flux boundary conditions. To verify these equations, it suffices to consider test-functions $\varphi_{j} \in$ $C_{c}^{\infty}\left((0, T) \times\left(\Omega \cup \Gamma_{j}\right)\right)$. We start from the ordinary differential equation (2.3) of the Galerkin scheme and the elliptic equations (2.4) to write

$$
\begin{aligned}
& \int_{0}^{T} \int_{\Omega} \partial_{t} s^{h} \varphi_{1} d x d t+\int_{0}^{T} \int_{\Omega} k_{1}\left(s^{h}, x\right)\left(\nabla \tilde{p}_{1}^{h}+g_{1}\right) \nabla \varphi_{1} d x d t \\
& \quad=-\int_{0}^{T} \int_{\Omega}\left[\Psi_{h}^{\tau}\left(p_{c}^{h}\left(s^{h}, x\right)-p^{h}\right)-\Psi_{h}^{\tau}\left(p_{c}\left(s^{h}, x\right)-p^{h}\right)\right] \varphi_{1} d x d t .
\end{aligned}
$$

As seen already in the proof of Lemma 2.4, the Lipschitz continuity of $p_{c}$ guarantees that the right hand side tends to zero as $h \rightarrow 0$. On the left hand side we can pass to the limit functions thanks to (2.24)-(2.26). This shows that the evolution equation (1.1) holds with the no-flux condition.

Equation (2.3) of the Galerkin scheme can also be combined with (2.5), tested with $\varphi_{2}$. The result is a relation similar to the above, but expressing $\partial_{t} s^{h}$ in terms of $k_{2}$ and $\tilde{p}_{2}$. The limit can be performed in the same way and provides the second evolution equation, (1.2).

Item 2. We want to show the pointwise inclusion for $p^{h}=p_{1}^{h}-p_{2}^{h}$ as demanded in item 2 of Definition 1.2. The discrete hysteresis system (2.7) provides, pointwise in $\Omega \times(0, T)$,

$$
\left[-\gamma^{h}(x), \gamma^{h}(x)\right] \ni p^{h}(x, t)-p_{c}^{h}\left(s^{h}(x, t), x\right)-\tau \partial_{t} s^{h}(x, t)
$$

for almost every $x \in \Omega$ and $t \in(0, T)$. Introducing small error terms, we write this relation as

$$
\begin{aligned}
{[-\gamma(x), \gamma(x)] \ni } & \tilde{p}^{h}(x, t)-p_{c}\left(s^{h}(x, t), x\right)-\tau \partial_{t} s^{h}(x, t) \\
& +\left(p^{h}-\tilde{p}^{h}\right)(x, t)+\left(p_{c}\left(s^{h}(x, t), x\right)-p_{c}^{h}\left(s^{h}(x, t), x\right)\right)+r^{h}(x, t),
\end{aligned}
$$

where the error term $r^{h}(x, t)$ concerns the replacement of $\gamma$ by $\gamma^{h}$ and satisfies $\left|r^{h}(x, t)\right| \leq\left|\gamma^{h}(x)-\gamma(x)\right| \leq C h$ due to the Lipschitz continuity of $\gamma$ in $x$. Similarly, the error introduced by $p_{c}\left(s^{h}\right)-p_{c}^{h}\left(s^{h}\right)$ is uniformly bounded by $h$.

Since $[-\gamma(x), \gamma(x)] \subset \mathbb{R}$ is a convex set, the set of functions $f: \Omega_{T} \rightarrow \mathbb{R}$ with $f(x, t) \in[-\gamma(x), \gamma(x)]$ is convex. As a convex and closed subset of $L^{2}\left(\Omega_{T}\right)$, it is also weakly closed. The right hand side of $(2.28)$ converges weakly in $L^{2}\left(\Omega_{T}\right)$ to $p-p_{c}(s)-\tau \partial_{t} s$, therefore this limit satisfies again the pointwise inclusion.

Item 3. We have to prove the variational inequality (1.12) for $\left(s, p_{1}, p_{2}\right)$. To this end we multiply equation (2.4) with $\tilde{p}_{1}^{h}-p_{0,1}$ and equation (2.5) with $\tilde{p}_{2}^{h}-p_{0,2}$ and 
integrate over $(0, T) \times \Omega$. We find

$$
\begin{aligned}
0= & \int_{0}^{T} \int_{\Omega} k_{1}\left(s^{h}, x\right)\left(\nabla \tilde{p}_{1}^{h}+g_{1}\right)\left(\nabla \tilde{p}_{1}^{h}-\nabla p_{0,1}\right) d x d t \\
& -\int_{0}^{T} \int_{\Omega} \Psi_{h}^{\tau}\left(p_{c}\left(s^{h}, x\right)-p_{1}^{h}+p_{2}^{h}\right)\left(\tilde{p}_{1}^{h}-p_{0,1}\right) d x d t
\end{aligned}
$$

and

$$
\begin{aligned}
0= & \int_{0}^{T} \int_{\Omega} k_{2}\left(s^{h}, x\right)\left(\nabla \tilde{p}_{2}^{h}+g_{2}\right)\left(\nabla \tilde{p}_{2}^{h}-\nabla p_{0,2}\right) d x d t \\
& +\int_{0}^{T} \int_{\Omega} \Psi_{h}^{\tau}\left(p_{c}\left(s^{h}, x\right)-p_{1}^{h}+p_{2}^{h}\right)\left(\tilde{p}_{2}^{h}-p_{0,2}\right) d x d t
\end{aligned}
$$

Adding these two equations yields

$$
\begin{aligned}
0= & \sum_{j=1}^{2}\left[\int_{0}^{T} \int_{\Omega} k_{j}\left(s^{h}, x\right)\left(\nabla \tilde{p}_{j}^{h}+g_{j}\right)\left(\nabla \tilde{p}_{j}^{h}-\nabla p_{0, j}\right)\right] d x d t \\
& -\int_{0}^{T} \int_{\Omega} \Psi_{h}^{\tau}\left(p_{c}\left(s^{h}, x\right)-p^{h}\right)\left(\tilde{p}^{h}-p_{0}\right) d x d t .
\end{aligned}
$$

The rest of this proof consists in performing the limit $h \rightarrow 0$ in (2.29). The limiting relation will be the variational inequality (1.12).

We start with some lower order terms. The weak convergences (2.24)-(2.25) and the strong convergence (2.26) together with the continuity of $k_{j}$ allow to take the limits

$$
\begin{gathered}
-\int_{0}^{T} \int_{\Omega} k_{j}\left(s^{h}, x\right)\left(\nabla \tilde{p}_{j}^{h}+g_{j}\right) \nabla p_{0, j} d x d t \rightarrow-\int_{0}^{T} \int_{\Omega} k_{j}(s, x)\left(\nabla p_{j}+g_{j}\right) \nabla p_{0, j} d x d t \\
\int_{0}^{T} \int_{\Omega} k_{j}\left(s^{h}, x\right) g_{j} \nabla \tilde{p}_{j}^{h} d x d t \rightarrow \int_{0}^{T} \int_{\Omega} k_{j}(s, x) g_{j} \nabla p_{j} d x d t .
\end{gathered}
$$

Concerning the quadratic term, we can use lower semi-continuity of the norm. Strong convergence of $s^{h}$ together with the continuity of $k_{j}$, using an argument based on Egorov's Theorem, provides

$$
\liminf _{h \rightarrow 0} \int_{0}^{T} \int_{\Omega} k_{j}\left(s^{h}, x\right)\left|\nabla \tilde{p}_{j}^{h}\right|^{2} d x d t \geq \int_{0}^{T} \int_{\Omega} k_{j}(s, x)\left|\nabla p_{j}\right|^{2} d x d t .
$$

We finally consider the terms in (2.29) containing $\Psi_{h}^{\tau}$. We exploit the Galerkin relation (2.3) in order to re-write the remaining term as

$$
\begin{aligned}
& -\int_{0}^{T} \int_{\Omega} \Psi_{h}^{\tau}\left(p_{c}\left(s^{h}\right)-p^{h}\right)\left(\tilde{p}^{h}-p_{0}\right) d x d t=\int_{0}^{T} \int_{\Omega} \partial_{t} s^{h}\left(\tilde{p}^{h}-p_{0}\right) d x d t \\
& +\int_{0}^{T} \int_{\Omega}\left[\Psi_{h}^{\tau}\left(p_{c}^{h}\left(s^{h}\right)-p^{h}\right)-\Psi_{h}^{\tau}\left(p_{c}\left(s^{h}\right)-p^{h}\right)\right]\left(\tilde{p}^{h}-p_{0}\right) d x d t .
\end{aligned}
$$


As noted before, the last integral of (2.30) tends to zero by the Lipschitz continuity of $p_{c}$ in $x$. In the other integral, the convergence

$$
-\int_{0}^{T} \int_{\Omega} \partial_{t} s^{h} p_{0} d x d t \rightarrow-\int_{0}^{T} \int_{\Omega} \partial_{t} s p_{0} d x d t
$$

is an immediate consequence of the weak convergence of $\partial_{t} s^{h}$.

In the remaining integral on the right hand side of (2.30) we use the hysteresis relation $(2.7)$,

$$
\begin{aligned}
& \int_{0}^{T} \int_{\Omega} \partial_{t} s^{h} \tilde{p}^{h} d x d t=\int_{0}^{T} \int_{\Omega} \partial_{t} s^{h} p^{h} d x d t \\
& \quad \in \int_{0}^{T} \int_{\Omega} \partial_{t} s^{h}\left(\Phi_{h}^{0}\left(\partial_{t} s^{h}\right)+\tau \partial_{t} s^{h}+p_{c}^{h}\left(s^{h}, x\right)\right) d x d t \\
& \quad=\int_{0}^{T} \int_{\Omega} \gamma^{h}(x)\left|\partial_{t} s^{h}\right|+\tau\left|\partial_{t} s^{h}\right|^{2}+\partial_{t} s^{h} p_{c}^{h}\left(s^{h}\right) d x d t .
\end{aligned}
$$

In the first two terms, the limit can be estimated by the weak lower semicontinuity of the $L^{2}$-norm,

$$
\liminf _{h \rightarrow 0} \int_{0}^{T} \int_{\Omega} \gamma^{h}(x)\left|\partial_{t} s^{h}\right|+\tau\left|\partial_{t} s^{h}\right|^{2} d x d t \geq \int_{0}^{T} \int_{\Omega} \gamma(x)\left|\partial_{t} s\right|+\tau\left|\partial_{t} s\right|^{2} d x d t .
$$

We exploited here the uniform convergence $\gamma^{h} \rightarrow \gamma$ on $\Omega$, which is a consequence of the Lipschitz continuity of $\gamma$.

The remaining integral over $\partial_{t} s^{h} p_{c}^{h}\left(s^{h}, x\right)$ is a total time derivative, but this fact is not needed here. We use the weak convergence of the first factor and the strong convergence of the second factor (note that $p_{c}^{h}$ is Lipschitz continuous in $s$ ) in order to conclude

$$
\int_{0}^{T} \int_{\Omega} \partial_{t} s^{h} p_{c}^{h}\left(x, s^{h}\right) d x d t \rightarrow \int_{0}^{T} \int_{\Omega} \partial_{t} s p_{c}(x, s) d x d t
$$

as $h$ tends to 0 . With this, we have analyzed all limits of integrals on the right hand side of (2.30), and thus of all integrals in (2.29). The variational inequality (1.12) is derived.

Lemma 2.7 provides the existence of a variational weak solution. By Lemma 1.3, this variational weak solution is a solution of the original problem as described in Theorem 1.1. Therefore the existence of a solution to the two-phase problem with hysteresis is shown.

\section{$3 \quad$ Numerical treatment}

We propose a straightforward generalization of the numerical scheme presented in [17]. We introduce $\Phi_{\delta}^{\tau}$ as a regularization of $\Phi^{\tau}$ in (2.1) with a positive regularizing parameter $\delta$, see (3.4), the inverse is denoted by $\Psi_{\delta}^{\tau}: \mathbb{R} \rightarrow \mathbb{R}$, see (3.5). We assume 
here that $\gamma>0$ and $\tau>0$ are constant. The two-phase system we consider then reads

$$
\begin{aligned}
\partial_{t} s & =\nabla \cdot\left(k_{1}(s)\left(\nabla p_{1}+\varrho_{1} e_{n}\right)\right), \quad(x, t) \in \Omega \times\left(t_{0}, T\right), \\
-\partial_{t} s & =\nabla \cdot\left(k_{2}(s)\left(\nabla p_{2}+\varrho_{2} e_{n}\right)\right), \quad(x, t) \in \Omega \times\left(t_{0}, T\right), \\
\partial_{t} s & =\Psi_{\delta}^{\tau}\left(p_{1}-p_{2}-p_{c}(s)\right), \quad(x, t) \in \Omega \times\left(t_{0}, T\right) .
\end{aligned}
$$

The functions $\Phi_{\delta}^{\tau}$ and $\Psi_{\delta}^{\tau}$ read

$$
\Phi_{\delta}^{\tau}=\Phi_{\delta}^{\tau}(\sigma)= \begin{cases}\gamma+\tau \sigma & \text { for } \quad \sigma>\delta, \\ \left(\frac{\gamma}{\delta}+\tau\right) \sigma & \text { for } \sigma \in[-\delta, \delta] \\ -\gamma+\tau \sigma & \text { for } \quad \sigma<-\delta,\end{cases}
$$

and

$$
\Psi_{\delta}^{\tau}=\Psi_{\delta}^{\tau}(z)= \begin{cases}\frac{z-\gamma}{\tau} & \text { for } z>\gamma+\tau \delta \\ \left(\frac{\gamma}{\delta}+\tau\right)^{-1} z & \text { for } z \in[-(\gamma+\tau \delta), \gamma+\tau \delta] \\ \frac{z+\gamma}{\tau} & \text { for } z<-(\gamma+\tau \delta) .\end{cases}
$$

In all numerical experiments, we use the permeabilities

$$
k_{1}(s)= \begin{cases}\kappa_{1} & \text { for } \quad s<a_{1} \\ \kappa_{1}+k_{0,1}\left(s-a_{1}\right)^{2} & \text { for } \quad s \geq a_{1}\end{cases}
$$

and

$$
k_{2}(s)= \begin{cases}\kappa_{2} & \text { for } \quad s>1-a_{2}, \\ \kappa_{2}+k_{0,2}\left(\left(1-a_{2}\right)-s\right)^{2} & \text { for } \quad s \leq 1-a_{2}\end{cases}
$$

with $a_{1}, a_{2} \in[0,1]$ and $\kappa_{1}, \kappa_{2}, k_{0,1}, k_{0,2}>0$. For $p_{c}$ we assume a van Genuchten type relation

$$
p_{c}(s)=\left\{\begin{array}{l}
\alpha_{+}\left(\frac{1}{1-a_{2}-s+\varepsilon_{p}}-\frac{1}{1-a_{2}-a_{1}+\varepsilon_{p}}\right) \\
\alpha_{-}\left(s-a_{1}\right) \quad \text { for } s \leq a_{1}
\end{array} \quad \text { for } s>a_{1},\right.
$$

with $\alpha_{ \pm} \in \mathbb{R}$ and a small regularizing parameter $\varepsilon_{p}>0$. For plots of the previously defined functions see Fig. 1.
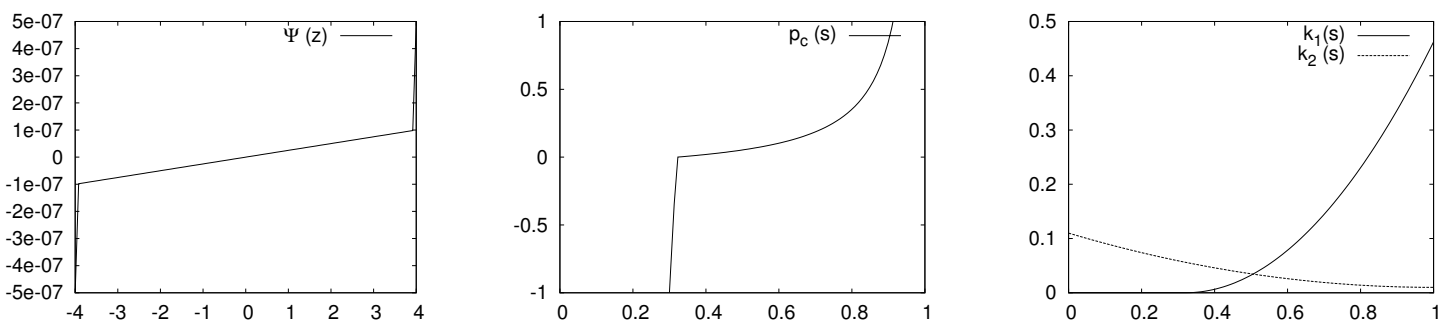

Figure 1: Plots of the functions $\Psi_{\delta}^{\tau}, p_{c}, k_{1}, k_{2}$ for parameters $\gamma=4, \tau=0.1$, $\delta=10^{-7}, \kappa_{1}=10^{-4}, \kappa_{2}=10^{-2}, k_{0,1}=1, k_{0,2}=0.1, a_{1}=0.32, a_{2}=0, \alpha_{+}=0.1$, $\alpha_{-}=50, \varepsilon_{p}=10^{-10}$.

For the numerical results presented here, we consider domains $\Omega:=(-L, L)^{n} \subset$ $\mathbb{R}^{n}$ with $L>0$. With the definition $\Gamma_{ \pm}:=\left\{x \in \bar{\Omega}: x_{n}= \pm L\right\} \subset \partial \Omega$ and given 
functions $p_{1,-}, j_{2,-}: \Gamma_{-} \times\left(t_{0}, T\right] \rightarrow \mathbb{R}$ and $j_{1,+}, p_{2,+}: \Gamma_{+} \times\left(t_{0}, T\right] \rightarrow \mathbb{R}$, we assume Dirichlet boundary conditions $p_{1}=p_{1,-}$ for $x \in \Gamma_{-}$and $p_{2}=p_{2,+}$ for $x \in \Gamma_{+}$and Neumann boundary conditions

$$
\begin{array}{ll}
j_{1}:=-k_{1}(s)\left(\nabla p_{1}+\varrho_{1} e_{n}\right) \cdot \nu_{+}=j_{1,+} & \text { for } \quad x \in \Gamma_{+}, \\
j_{2}:=-k_{2}(s)\left(\nabla p_{2}+\varrho_{2} e_{n}\right) \cdot \nu_{-}=j_{2,-} & \text { for } \quad x \in \Gamma_{-},
\end{array}
$$

$\nu_{ \pm}= \pm e_{n}$ denoting the outer normals to $\Gamma_{ \pm}$. In the lateral directions, i.e. for $x_{i} \in\{-L, L\}, i \in\{1, \ldots, n-1\}$, we assume periodic pressures $p_{1}$ and $p_{2}$.

\subsection{Discretization}

The numerical approach is to discretize the Galerkin scheme of Definition 2.1 and to regard the corresponding set of equations (for every time step) as one large system for saturation and pressures. All nonlinear terms are linearized in the same way as proposed in [17].

In space, we apply linear finite elements to discretize the above system. We apply adaptivity in space with an $L^{2}$-like error indicator for the discrete saturation based on the jump residual as discussed in [17]. Furthermore, we use a simple adaptive strategy in time, where the time step $\Delta t_{m}$ is inversely proportional to the maximum of the discrete time derivative of the saturation [17]. We present numerical examples which show the validity of the algorithm implemented in the FEM toolbox AMDiS [27]. The resulting linear system of equations is solved by a direct solver (UMFPACK, $[15])$.

\subsection{Numerical Results}

For the numerical results presented in the following, we have used a time dependent boundary flux

$$
j_{1,+}= \begin{cases}j_{1,+}^{0} & \text { for } t<t_{s}, \\ j_{1,+}^{s} & \text { for } t \geq t_{s}\end{cases}
$$

with $t_{s}>t_{0}$ and $j_{1,+}^{0}, j_{1,+}^{s} \in \mathbb{R}$. The change in the upper boundary condition at a switching time $t_{s}$ is important in the modelling of fingering experiments, see [24]. On the lower boundary, we assume the constant flux

$$
j_{2,-} \equiv j_{2,-}^{0}
$$

for a given value $j_{2,-}^{0} \in \mathbb{R}$. The Dirichlet boundary conditions are

$$
p_{1,-} \equiv \gamma-\alpha_{-} a_{1}, \quad p_{2,+} \equiv p_{2,+}^{0}
$$

with a constant pressure $p_{2,+}^{0}$. We study perturbations of the initial condition $s=0$ of the form

$$
s_{0}(x)=\sum_{i=1}^{10} A_{i}\left(1-\tanh \left(3\left(\left|x-x_{0, i}\right|-1 / 2\right)\right)\right),
$$

where $x_{0, i}=\left(-L+\frac{(2 i-1)}{10} L, 23.5\right), i=1, \ldots, 10$ and amplitudes $A_{1}=0.6, A_{2}=0.4$, $A_{3}=0.2, A_{4}=0.5, A_{5}=0.2, A_{6}=0.3, A_{7}=0.1, A_{8}=0.7, A_{9}=0.5, A_{10}=0.1$. We provide a list of all other parameters in Table 1. 


\begin{tabular}{|c|c|c|c|c|c|c|c|c|c|c|}
\hline parameter & $\gamma$ & $\kappa_{1}$ & $\kappa_{2}$ & $k_{0,1}$ & $k_{0,2}$ & $\delta$ & $\tau$ & $a_{1}$ & $a_{2}$ & \\
\hline value & 4 & $10^{-4}$ & $10^{-2}$ & 1 & 0.1 & $10^{-7}$ & $0.1 ; 0.5$ & 0.32 & 0 & \\
\hline \hline parameter & $\varrho_{1}$ & $\varrho_{2}$ & $\varepsilon_{p}$ & $\alpha_{+}$ & $\alpha_{-}$ & $j_{1,+}^{0}$ & $j_{1,+}^{s}$ & $p_{2,+}^{0}$ & $j_{2,-}^{0}$ & $L$ \\
\hline value & 1 & 0 & $10^{-10}$ & 0.1 & 50 & 0.524 & 0.01 & 0 & 0 & 24 \\
\hline
\end{tabular}

Table 1: Parameters used for numerical results.

In the following, we will compare numerical results for the two-phase system with results for the hysteresis Richards system which is solved by the numerical method described in [17]. In order to be able to compare the solutions at similar times $t$, we have chosen a time-dependent parameter

$$
\kappa_{2}=\kappa_{2}(t)=\left\{\begin{array}{l}
10^{5} \quad \text { for } t<t_{s} \\
10^{-2} \text { for } t \geq t_{s}
\end{array}\right.
$$

Formally, in the limit $\kappa_{2} \rightarrow \infty$, the two-phase system reduces to the hysteresis Richards model treated in [17]. The above choice of $\kappa_{2}$ has the effect that the discrete saturation fields for the two models are almost identical at time $t=t_{s}$. We emphasize that, also after the switching time $t_{s}$, in regions of low saturation, the permeability of the second fluid is much larger than the permeability of the first fluid, see Figure 1 .

\subsubsection{First results for two-phase flow evolution}

Numerical results for the evolution of fingers in the two-phase flow model are presented in Fig. 2. The gray-scale picture indicates the saturation $s_{h}$ for the parameter $\tau=0.5$ and the initial condition (3.8). From the perturbations, fingers start to grow and evolve basically into the direction of gravity.
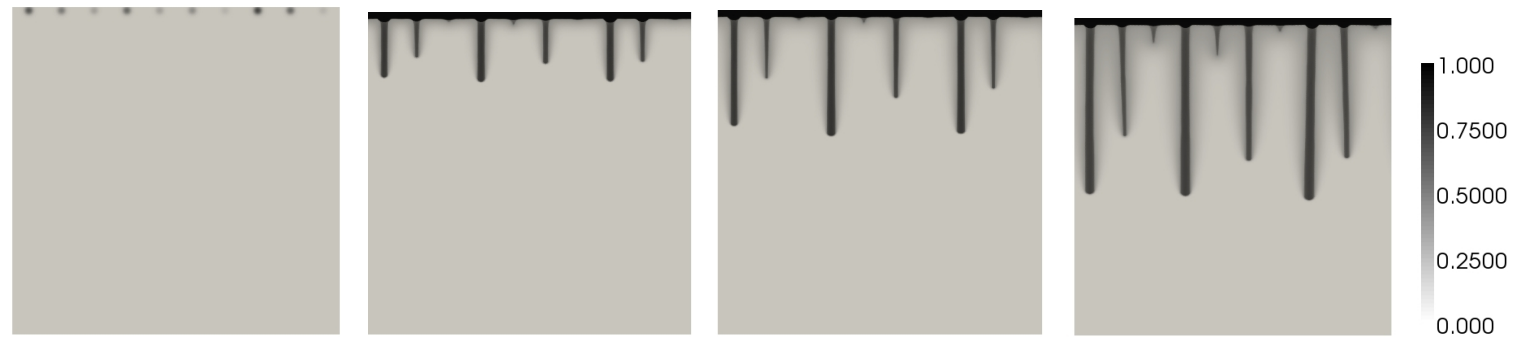

Figure $2:$ Discrete saturation for $\tau=0.5$ at times $t=-2, t \approx 106, t \approx 205, t \approx 421$.

We observe that fingers can develop in the two-phase flow system with hysteresis. Some fingers seize to grow after some time, the surviving fingers have a comparable length, but there are differences in width and length. We also observe that fingers can repel each other, we refer to the last two long fingers in Figure 2.

We recall that the hysteresis terms contribute only in an indirect way to the evolution of fingers. Below the highly wetted top layer of the domain, the system is in an imbibition process, hence the static hysteresis is not of relevance in that region. In contrast, in the top layer of the domain, the effect of the hysteresis terms is that the variations of the saturation distribution cannot be removed during the evolution. 


\subsubsection{Comparison with the Richards equation}

Our aim is to compare numerically the two-phase flow equations with the Richards equation. More precisely, we investigate if the presence of a second fluid has an effect that can be compared to the effect of variations in the time-delay parameter $\tau$. For this study, we compare four different solutions as indicated in Table 2.

\begin{tabular}{|c|c|}
\hline $\begin{array}{c}\text { two-phase flow, } \tau=0.5 \\
\text { Fig. 3, left }\end{array}$ & $\begin{array}{c}\text { Richards equation, } \tau=0.5 \\
\text { Fig. 3, middle }\end{array}$ \\
\hline $\begin{array}{c}\text { two-phase flow, } \tau=0.1 \\
\text { Fig. 4, left }\end{array}$ & $\begin{array}{c}\text { Richards equation, } \tau=0.1 \\
\text { Fig. 4, middle }\end{array}$ \\
\hline
\end{tabular}

Table 2: Description of the data set generating four different solutions.

Three qualitative observations can be made. First, the deviations of the growth directions from the direction of gravity are present for several fingers in the case of two-phase flow, but not for the Richards equation. Second, for the two-phase flow system, fingers tend to be thicker. Third, long fingers are shorter and short fingers are longer, if compared to the results for the Richards equation. These differences are further illustrated in the right part of Fig. 3, where level sets $\left\{s_{h}=\frac{1}{2}\right\}$ are displayed in one plot for both cases.
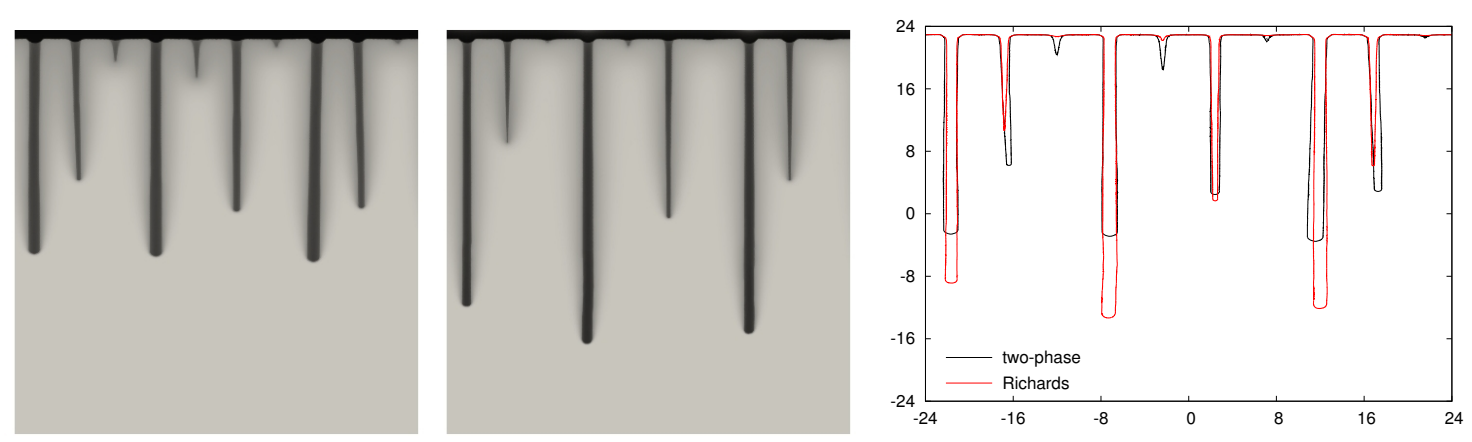

Figure 3: Discrete saturation for two-phase flow (left) and Richards equation (middle) at time $t \approx 421$, plot of level sets $\left\{s_{h}=\frac{1}{2}\right\}$ (right, black: two-phase; red/gray: Richards equation). In both cases $\tau=0.5$ has been used.

A similar comparison is shown in Fig. 4 for $\tau=0.1$. Basically, the same qualitative differences between two-phase flow and Richards equation are visible. In addition, in both cases, reduction of $\tau$ corresponds to reduction of the thickness of the fingers.

We conclude with the observation that in the parameter setup investigated here, the influence of including a second phase in the model is not comparable with the influence of reducing the parameter $\tau$.

\section{Acknowledgement}

The authors gratefully acknowledge the support by DFG-grant SCHW 639/3-1. 

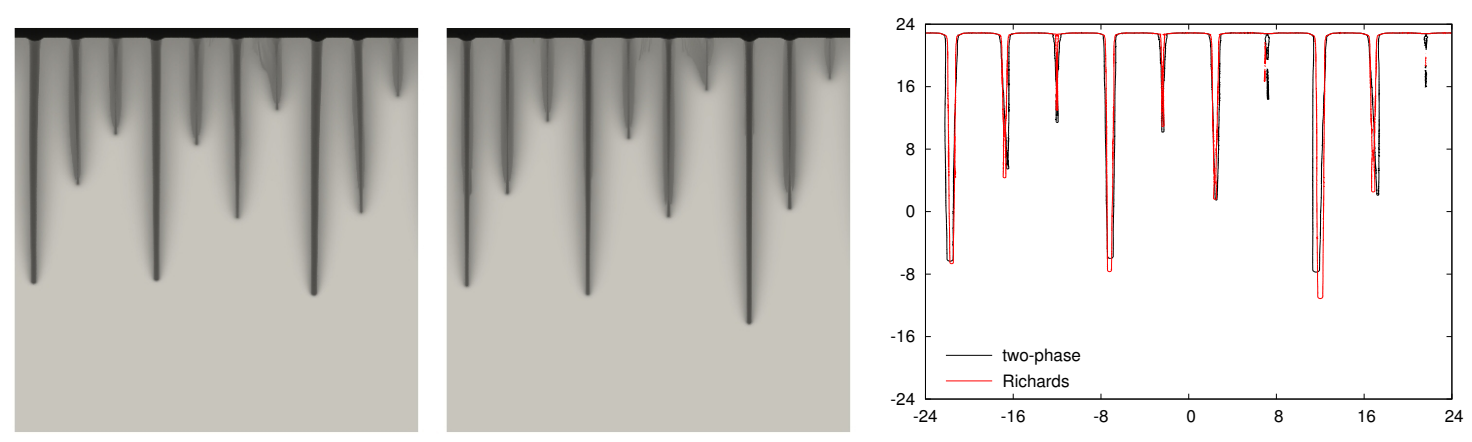

Figure 4: Discrete saturation for two-phase flow (left) and Richards equation (middle) at times $t \approx 529$ and $t \approx 528$, respectively, plot of level sets $\left\{s_{h}=\frac{1}{2}\right\}$ (right, black: two-phase; red/gray: Richards equation). In both cases $\tau=0.1$ has been used.

\section{References}

[1] H. W. Alt and E. DiBenedetto. Nonsteady flow of water and oil through inhomogeneous porous media. Ann. Scuola Norm. Sup. Pisa Cl. Sci. (4), 12(3):335-392, 1985.

[2] H. W. Alt and S. Luckhaus. Quasilinear elliptic-parabolic differential equations. Math. Z., 183(3):311-341, 1983.

[3] H. W. Alt, S. Luckhaus, and A. Visintin. On nonstationary flow through porous media. Ann. Mat. Pura Appl. (4), 136:303-316, 1984.

[4] T. Arbogast, M. F. Wheeler, and N.-Y. Zhang. A nonlinear mixed finite element method for a degenerate parabolic equation arising in flow in porous media. SIAM J. Numer. Anal., 33(4):1669-1687, 1996.

[5] F. Bagagiolo and A. Visintin. Hysteresis in filtration through porous media. Z Anal. Anwendungen, 19(4):977-997, 2000.

[6] F. Bagagiolo and A. Visintin. Porous media filtration with hysteresis. Adv. Math. Sci. Appl., 14(2):379-403, 2004.

[7] P. Bastian and R. Helmig. Efficient fully-coupled solution techniques for twophase flow in porous media. Advances in Water Resources, 23(3):199-216, 1999.

[8] A. Y. Beliaev and S. M. Hassanizadeh. A theoretical model of hysteresis and dynamic effects in the capillary relation for two-phase flow in porous media. Transp. Porous Media, 43(3):487-510, 2001.

[9] F. Buzzi, M. Lenzinger, and B. Schweizer. Interface conditions for degenerate two-phase flow equations in one space dimension. Analysis (Munich), 29(3):299$316,2009$.

[10] C. Cancès, C. Choquet, Y. Fan, and I. Pop. Existence of weak solutions to a degenerate pseudo-parabolic equation modeling two-phase flow in porous media. Technical report, Eindhoven University of Technology, CASA, 2010. 
[11] C. Cancès and M. Pierre. An existence result for multidimensional immiscible two-phase flows with discontinuous capillary pressure field. HAL: hal-00518219, 2011.

[12] J. Carrillo. On the uniqueness of the solution of the evolution dam problem. Nonlinear Anal., 22(5):573-607, 1994.

[13] Z. Chen. Degenerate two-phase incompressible flow. I. Existence, uniqueness and regularity of a weak solution. J. Differential Equations, 171(2):203-232, 2001.

[14] Z. Chen. Degenerate two-phase incompressible flow. II. Regularity, stability and stabilization. J. Differential Equations, 186(2):345-376, 2002.

[15] T. A. Davis. Algorithm 832: UMFPACK V4.3 - an unsymmetric-pattern multifrontal method. ACM Trans. Math. Software, 30(2):196-199, 2004.

[16] D. Kröner and S. Luckhaus. Flow of oil and water in a porous medium. J. Differential Equations, 55(2):276-288, 1984.

[17] A. Lamacz, A. Rätz, and B. Schweizer. A well-posed hysteresis model for flows in porous media and applications to fingering effects. Adv. Math. Sci. Appl., 21(1), 2011.

[18] M. Lenzinger and B. Schweizer. Two-phase flow equations with outflow boundary conditions in the hydrophobic-hydrophilic case. Nonlinear Anal., 73(4):840-853, 2010 .

[19] A. Mikelić. A global existence result for the equations describing unsaturated flow in porous media with dynamic capillary pressure. J. Differential Equations, 248(6):1561-1577, 2010.

[20] F. Otto. $L^{1}$-contraction and uniqueness for unstationary saturated-unsaturated porous media flow. Adv. Math. Sci. Appl., 7(2):537-553, 1997.

[21] F. Radu, I. S. Pop, and P. Knabner. Order of convergence estimates for an Euler implicit, mixed finite element discretization of Richards' equation. SIAM J. Numer. Anal., 42(4):1452-1478 (electronic), 2004.

[22] B. Schweizer. Averaging of flows with capillary hysteresis in stochastic porous media. European J. Appl. Math., 18(3):389-415, 2007.

[23] B. Schweizer. Regularization of outflow problems in unsaturated porous media with dry regions. J. Differential Equations, 237(2):278-306, 2007.

[24] B. Schweizer. Instability of gravity wetting fronts for richards equations with hysteresis. submitted to IFB, 2010.

[25] J. S. Selker, J.-Y. Parlange, and T. S. Steenhuis. Fingered flow in two dimensions. part 2. predicting finger moisture profile. Wat. Resources Res., 28(9):2523-2528, 1992. 
[26] C. J. van Duijn, G. J. M. Pieters, and P. A. C. Raats. Steady flows in unsaturated soils are stable. Transp. Porous Media, 57(2):215-244, 2004.

[27] S. Vey and A. Voigt. AMDiS - adaptive multidimensional simulations. Comput. Visual. Sci., 10:57-67, 2007.

[28] A. Visintin. Differential models of hysteresis, volume 111 of Applied Mathematical Sciences. Springer-Verlag, Berlin, 1994. 


\section{Preprints ab 2009/12}

2011-12

2011-11

2011-10

2011-09

2011-08

2011-07

2011-06

2011-05

2011-04

2011-03

2011-02

2011-01

2010-16

$2010-15$

2010-14

2010-13

2010-12
J. Koch, A. Rätz, and B. Schweizer

Two-phase flow equations with a dynamic capillary pressure

Michael Voit

Central limit theorems for hyperbolic spaces and Jacobi processes on $[0, \infty[$

Ben Schweizer

The Richards equation with hysteresis and degenerate capillary pressure

Andreas Rätz and Matthias Röger

Turing instabilities in a mathematical model for signaling networks

Matthias Röger and Reiner Schätzle

Control of the isoperimetric deficit by the Willmore deficit

Frank Klinker

Generalized duality for k-forms

Sebastian Aland, Andreas Rätz, Matthias Röger, and Axel Voigt

Buckling instability of viral capsides - a continuum approach

Wilfried Hazod

The concentration function problem for locally compact groups revisited: Non-dissipating space-time random walks, $\tau$-decomposable laws and their continuous time analogues

Wilfried Hazod, Katrin Kosfeld

Multiple decomposability of probabilities on contractible locally compact groups

Alexandra Monzner* and Frol Zapolsky $\dagger$

A comparison of symplectic homogenization and Calabi quasi-states

Stefan Jäschke, Karl Friedrich Siburg and Pavel A. Stoimenov

Modelling dependence of extreme events in energy markets using tail copulas

Ben Schweizer and Marco Veneroni

The needle problem approach to non-periodic homogenization

Sebastian Engelke and Jeannette H.C. Woerner

A unifying approach to fractional Lévy processes

Alexander Schnurr and Jeannette H.C. Woerner

Well-balanced Lévy Driven Ornstein-Uhlenbeck Processes

Lorenz J. Schwachhöfer

On the Solvability of the Transvection group of Extrinsic

Symplectic Symmetric Spaces

\section{Marco Veneroni}

Stochastic homogenization of subdifferential inclusions via scale integration

Agnes Lamacz, Andreas Rätz, and Ben Schweizer

A well-posed hysteresis model for flows in porous media and

applications to fingering effects 
Confined elastic curves

2010-09

Matthias Röger and Hendrik Weber

Tightness for a stochastic Allen-Cahn equation

2010-08

\section{Michael Voit}

Multidimensional Heisenberg convolutions and product formulas

for multivariate Laguerre polynomials

Ben Schweizer

Instability of gravity wetting fronts for Richards equations with hysteresis

2010-06

Lorenz J. Schwachhöfer

Holonomy Groups and Algebras

2010-05

Agnes Lamacz

Dispersive effective models for waves in heterogeneous media

Ben Schweizer and Marco Veneroni

Periodic homogenization of Prandtl-Reuss plasticity equations in arbitrary dimension

A copula-based nonparametric measure of regression dependence

2010-02

René L. Schilling and Alexander Schnurr

The Symbol Associated with the Solution of a Stochastic Differential Equation

2010-01

Henryk Zähle

Rates of almost sure convergence of plug-in estimates for distortion risk measures

Lorenz J. Schwachhöfer

Nonnegative curvature on disk bundles

2009-15 Iuliu Pop and Ben Schweizer

Regularization schemes for degenerate Richards equations and outflow conditions

2009-14 Guy Bouchitté and Ben Schweizer

Cloaking of small objects by anomalous localized resonance

2009-13 Tom Krantz, Lorenz J. Schwachhöfer

Extrinsically Immersed Symplectic Symmetric Spaces

2009-12

\section{Alexander Kaplun}

Continuous time Ehrenfest process in term structure modelling 